ESAIM: PROCEEDINGS, September 2005, Vol.14, 174-200

Eric Cancès \& Jean-Frédéric Gerbeau, Editors

DOI: $10.1051 /$ proc:2005014

\title{
PARAMETER IDENTIFICATION FOR A ONE-DIMENSIONAL BLOOD FLOW MODEL *
}

\author{
Vincent Martin $^{1}$, Francois Clément $^{2}$, Astrid Decoene $^{3}$ And Jean-Frédéric \\ GERBEAU $^{4}$
}

\begin{abstract}
The purpose of this work is to use a variational method to identify some of the parameters of one-dimensional models for blood flow in arteries. These parameters can be fit to approach as much as possible some data coming from experimental measurements or from numerical simulations performed using more complex models.

A nonlinear least squares approach to parameter estimation was taken, based on the optimization of a cost function. The resolution of such an optimization problem generally requires the efficient and accurate computation of the gradient of the cost function with respect to the parameters.

This gradient is computed analytically when the one-dimensional hyperbolic model is discretized with a second order Taylor-Galerkin scheme. An adjoint approach was used.

Some preliminary numerical tests are shown. In these simulations, we mainly focused on determining a parameter that is linked to the mechanical properties of the arterial walls, the compliance. The synthetic data we used to estimate the parameter were obtained from a numerical computation performed with a more accurate model: a three-dimensional fluid-structure interaction model. The first results seem to be promising. In particular, it is worth noticing that the estimated compliance which gives the best fit is quite different from the values that are commonly used in practice.
\end{abstract}

Résumé. Le but de ce travail est d'identifier certains des paramètres existant dans des modèles 1-d d'écoulement sanguin dans des artères. Ces paramètres peuvent permettre d'approcher autant que possible des configurations géométriques réalistes ou des données expérimentales. Une approche de l'estimation de paramètres par moindres carrés non-linéaires a été adoptée, basée sur l'optimisation d'une certaine fonction coût. La résolution d'un tel problème de minimisation requiert le calcul efficace et précis du gradient de la fonction coût par rapport aux paramètres. Le gradient est discrétisé analytiquement dans le cas d'une discrétisation du modèle hyperbolique 1-d par le schema de TaylorGalerkin. Une approche par l'état adjoint a été employée.

Des premiers résultats numériques sont fournis. Pour ces simulations, nous nous sommes concentrés sur la détermination d'un paramètre lié aux propriétés mécaniques de la paroi artérielle. Les données synthétiques utilisées pour l'estimation de ce paramètre ont été obtenues à partir d'un modèle beaucoup plus raffiné : un modèle 3 -d d'interaction fluide-structure. Les résultats semblent intéressants car le paramètre estimé est assez différent de ce à quoi on s'attendrait a priori.

\footnotetext{
* This study was supported by RTN-Project "HaeMOdel", project no HPRN-CT-2002-00270.

1 MOX, Dpto di Matematica, Politecnico di Milano, Via Bonardi 9, 20133 Milano, Italy ;

e-mail: vincent.martin@mate.polimi.it \& vincent.martin@inria.fr

2 Estime, Inria Rocquencourt, BP 105, 78153 Le Chesnay, France ; e-mail: francois.clement@inria.fr

3 Bang, Inria Rocquencourt, BP 105, 78153 Le Chesnay, France ; e-mail: astrid.decoene@inria.fr

4 Reo, Inria Rocquencourt, BP 105, 78153 Le Chesnay, France ; e-mail: jean-frederic.gerbeau@inria.fr
} 


\section{INTRODUCTION}

We focus in this study on the parameter estimation of a 1-d blood flow model, [11,19]:

$$
\begin{aligned}
\frac{\partial A}{\partial t}+\frac{\partial Q}{\partial z} & =0 \\
\frac{\partial Q}{\partial t}+\frac{\partial}{\partial z}\left(\frac{\alpha Q^{2}}{A}\right)+\frac{A}{\rho} \frac{\partial P}{\partial z}+K_{r}\left(\frac{Q}{A}\right) & =0
\end{aligned}
$$

where the pressure $P$, the area $A$ and the flux $Q$ are the unknowns of the problem. We denoted by $z$ the abscissa, $t$ the time, $\rho$ the density of the blood. Two parameters, the Coriolis coefficient $\alpha$ and the friction parameter $K_{r}$, are introduced in this model. This system is closed with a wall displacement law of the form

$$
P(t, z)-P_{e x t}=\tilde{\beta}\left(A^{1 / 2}-A_{0}^{1 / 2}\right),
$$

that links the pressure and the area. We introduced the external pressure $P_{\text {ext }}$, the area at rest $A_{0}$ and a coefficient $\tilde{\beta}$ that takes into account the mechanical characteristics of the arterial wall. The parameters $\left(\alpha, \tilde{\beta}, K_{r}, A_{0}\right)$ used in this model are related to physiological data or to the velocity profile. Thus our aim is to identify some of these parameters.

Motivations. Two main objectives can be thought of to motivate the parameter estimation in 1-d models. The first goal may have interesting clinical applications. Knowing some non-invasive clinical data measured on a patient, one would like to retrieve the actual physiological or mechanical constants of this patient. For instance, it is possible to measure unintrusively the mean fluxes and areas as a function of time at two or three different sections of an artery. From these data, one would like to identify the mechanical properties of the arterial wall: we could thus obtain the compliance of the wall and the pressure in a totally non-invasive manner.

The second objective is consists in making a coarsening of models. One is now able to solve the full 3-d fluid structure interaction problem on real geometries, coming from real patients. However the resolution of this problem is quite expensive and this complex model cannot probably be afforded for an intensive numerical study requiring lots of resolutions. This can occur when one wishes to modify the configuration of the flux or the boundary conditions for instance. In this case, a single resolution of the accurate but expensive 3-d model could provide data, such as the flux and the area for all sections of the mesh. Then one can estimate the 1-d parameters from the 3-d data; this would allow to make use of the cheap 1-d model for intensive computations, but using the parameters that take into account data coming from a real geometry and a physically more detailed model. This multiscale approach has already been used in a medical application: some 3-d NavierStokes (without compliant walls) were performed to obtain a simple numerical/experimental law that was used in ulterior 0 -d simulations, $[16,17]$. The difference is that in this study, we base our parameter identification on sound mathematical tools, and we focus on 1-d models that provide a more accurate description of wave propagation in the large arteries. One of the conclusions of this work is that the coefficient estimated by solving the inverse problem is quite different from the coefficient one would have chosen a priori (an a priori expression for $\tilde{\beta}$ is provided in Section 1). Thus this approach could provide 1-d models that are suitable for a 3-d-1-d coupling in multiscale computations, see [8]. This illustrates the relevance of our approach.

To conclude on the motivations, one can either aim at estimating physical parameters from experimental unintrusive measurements, or at having a cheap 1-d model to be as close as possible to an expensive 3-d model, in order to make realistic configuration studies using this cheap model. We note here that the methodology remains identical for both objectives, the only difference being the expression of the measurement operator.

Methodology. We followed a standard nonlinear least squares approach to parameter estimation, [3-5]. It is based on the optimization of an appropriate cost function. The resolution of such a minimization problem generally requires the efficient and accurate computation of the gradient of the cost function with respect to the parameters, [4]. We discretized the 1-d model with a second order Taylor-Galerkin scheme, [1]. We computed analytically the gradient of the discrete cost function, using an adjoint approach. The adjoint problem obtained 
is, as expected, a linear 1-d hyperbolic system, but has nonstandard discretization and boundary conditions, that are due to the differentiation of the Taylor-Galerkin scheme.

A previous attempt was made in [14] to estimate the elasticity of the arteries. However, instead of differentiating the discrete equations as we do here, the author differentiated first the continuous equations and then discretized the continuous adjoint problem thus obtained. We believe this is a possible reason for the very slow convergence he reached in the minimization process (about 1500 iterations for three parameters).

Finally, this approach allows to make a sensitivity analysis, $[13,15]$, that can provide information on the relevant parameters to estimate, and on the type of measurements to perform. But this goes beyond the scope of this study.

Results. The analytical discrete gradient was implemented and validated by comparison with finite differences approximations. The adjoint problem was not computed by automatic code differentiation, but directly implemented. Thus we could easily control the memory required by the gradient code. We used a constrained optimization code based on a quasi-Newton method with active constraints.

We present some preliminary numerical results. In these numerical simulations, we mainly focused on determining the parameter $\tilde{\beta}$ that is linked to the mechanical properties, i.e. the compliance, of the arterial walls. The synthetic data we used to estimate the parameter were obtained from a numerical computation performed with a 3-d fluid structure interaction model. We first used as data the values of the areas and fluxes at only two or three points of the domain (boundaries plus maybe the middle point). Although the data at two points do not seem to be enough to find a stable value, it seems that with three points, one can obtain a $\tilde{\beta}$ relatively stable (i.e. it is little changed when the estimation is made with all available spatial data). In the second numerical tests, we used all data available from the 3-d computation. These first numerical results seem promising and should be followed by further developments.

In Section 1, we present briefly the continuous 1-d model, that is derived in Section 2 with the Taylor-Galerkin scheme. In Section 3, the gradient of the least squares cost function is computed with the adjoint approach. Numerical results are presented in Section 4, and some conclusions and perspectives in Section 5.

\section{Direct MOdel: 1-D BloOd Flow MODEL}

We present in this section a 1-d blood flow model based on the works in [10,20]. See also [19]. It is a 1-d vectorial hyperbolic problem, with a $2 \times 2$ flux matrix that admits two real eigenvalues with opposite signs under physiological conditions.

We leave the problem of the parameterization and of the measurements for the next sections, see Section 3.1.

\subsection{Continuous blood flow model}

Let $\Omega=(0, L)$ be a 1-d domain of length $L>0$. Let $I=\left(0, T_{f}\right)$, with $T_{f}>0$, the time interval of simulation. The continuous system of equations reads, for the abscissa $z \in \Omega$ and the time $t \in I$

$$
\begin{aligned}
\frac{\partial A}{\partial t}+\frac{\partial Q}{\partial z} & =0, \quad z \in \Omega, \quad t \in I \\
\frac{\partial Q}{\partial t}+\frac{\partial}{\partial z}\left(\frac{\alpha Q^{2}}{A}\right)+\frac{A}{\rho} \frac{\partial P}{\partial z}+K_{r}\left(\frac{Q}{A}\right) & =0, \quad z \in \Omega, \quad t \in I
\end{aligned}
$$

where $\mathbf{U}=[A, Q]^{\top}$ is the vectorial unknown of the problem, made of the area $A$ and of the flux $Q$. The pressure $P$ is an intermediary unknown. We denoted by $\rho$ the blood density that we assume perfeclty known in this study. Two parameters, the Coriolis coefficient $\alpha$ and the friction parameter $K_{r}$, are introduced when deriving this model. This system of partial differential equations is completed with an initial condition

$$
\mathbf{U}(0, z)=\mathbf{U}_{\mathbf{0}}(z), \quad z \in \Omega,
$$


and some adapted boundary conditions

$$
\begin{aligned}
\phi_{0}(\mathbf{U}(t, 0), \mathbf{p}) & =q_{0}(t), \quad t \in I \\
\phi_{L}(\mathbf{U}(t, L), \mathbf{p}) & =q_{L}(t), \quad t \in I
\end{aligned}
$$

In equations (2) and (3), $\mathbf{U}_{\mathbf{0}}, q_{0}$ and $q_{L}$ are given initial and boundary data. The definition of the real-valued boundary functions $\phi_{0}, \phi_{L}$ is discussed in Section 2.2.

The system (1) is closed with a wall displacement law of the form, see [21] for instance and the reference therein,

$$
P(t, z)-P_{e x t}=\psi\left(A ; A_{0}, \boldsymbol{\beta}\right)=\beta_{0}\left[\left(\frac{A}{A_{0}}\right)^{\beta_{1}}-1\right]
$$

where $\boldsymbol{\beta}=\left(\beta_{0}, \beta_{1}\right)$ is a pair of positive real parameters. The power coefficient $\beta_{1}$ is often taken equal to $1 / 2$, which means that the pressure difference is proportional to the wall displacement, and, in this case, a linear elastic law can provide an expression for $\beta_{0}$, if the mechanical properties of the arterial wall are known $a$ priori,

$$
\beta_{1}=1 / 2, \quad \beta_{0}=\frac{\sqrt{\pi} h_{0, w} E}{\sqrt{A_{0}}\left(1-\nu^{2}\right)},
$$

where $h_{0, w}$ is the wall thickness, $E$ is the wall Young modulus, and $\nu$ is the Poisson coefficient. We can reformulate the wall displacement law in the following way:

$$
P(t, z)-P_{e x t}=\psi\left(A ; A_{0}, \beta\right)=2 \rho \beta\left[A^{1 / 2}-A_{0}^{1 / 2}\right], \quad \text { with } \quad \beta=\frac{\beta_{0}}{2 \rho A_{0}}=\frac{\sqrt{\pi} h_{0, w} E}{2 \rho A_{0}\left(1-\nu^{2}\right)}
$$

We introduce the following quantity

$$
c=c\left(A ; A_{0}, \boldsymbol{\beta}\right)=\sqrt{\frac{A}{\rho} \frac{\partial \psi}{\partial A}}=\sqrt{\frac{\beta_{0} \beta_{1}}{\rho}\left(\frac{A}{A_{0}}\right)^{\beta_{1}}}
$$

which has the dimension of a velocity and is related to the speed of propagation of simple waves along the tube. We also introduce the integral of the square of the celerity $c$ with respect to the area

$$
C\left(A ; A_{0}, \boldsymbol{\beta}\right)=\int_{A_{0}}^{A} c^{2}\left(\tau ; A_{0}, \boldsymbol{\beta}\right) d \tau=\frac{\beta_{0} \beta_{1} A_{0}}{\rho\left(\beta_{1}+1\right)}\left[\left(\frac{A}{A_{0}}\right)^{\beta_{1}+1}-1\right] .
$$

Defining the flux function

$$
\mathbf{F}(\mathbf{U}, \mathbf{p})=\left[\begin{array}{c}
Q \\
\alpha \frac{Q^{2}}{A}+C
\end{array}\right]
$$

and the source term

$$
\mathbf{B}(\mathbf{U}, \mathbf{p})=\left[\begin{array}{c}
0 \\
B_{2}
\end{array}\right], \quad B_{2}=K_{r} \frac{Q}{A}+\frac{A}{\rho} \frac{\partial \psi}{\partial A_{0}} \frac{d A_{0}}{d z}+\frac{A}{\rho} \frac{\partial \psi}{\partial \boldsymbol{\beta}} \frac{d \boldsymbol{\beta}}{d z}-\frac{\partial C}{\partial A_{0}} \frac{d A_{0}}{d z}-\frac{\partial C}{\partial \boldsymbol{\beta}} \frac{d \boldsymbol{\beta}}{d z},
$$

we can write the complete problem in a conservative form

$$
\frac{\partial \mathbf{U}}{\partial t}+\frac{\partial}{\partial z}[\mathbf{F}(\mathbf{U}, \mathbf{p})]+\mathbf{B}(\mathbf{U}, \mathbf{p}) \quad=\quad \mathbf{0}, \quad z \in \Omega \quad t \in I
$$


We introduce the jacobian matrix

$$
\mathbf{H}(\mathbf{U}, \mathbf{p})=\frac{\partial \mathbf{F}}{\partial \mathbf{U}}=\left[\begin{array}{cc}
0 & 1 \\
c^{2}-\alpha\left(\frac{Q}{A}\right)^{2} & 2 \alpha \frac{Q}{A}
\end{array}\right],
$$

whose eigenvalues are real, distinct, see [19]. It is also noted that for common values of the blood flow in human arteries, these eigenvalues have opposite signs. We assume in the rest of the article that this hypothesis holds.

Remark 1.1. We can make two remarks concerning the initial and boundary terms. First, when simulating a phenomenon that is periodic in time such as the arterial flow, the initial data $\mathbf{U}_{\mathbf{0}}$ should not interfere on the results. Therefore we did not consider it as one of the parameters to be estimated.

Second, as already noticed, the boundary conditions must be properly chosen. The number of boundary conditions to impose at each end of the vessel equals the number of characteristics entering the domain through that boundary. As the eigenvalues are distinct with opposite signs, i.e. the flow is sub-critical everywhere, one must impose exactly one scalar boundary condition at $z=0$ and $z=L$, see [19].

Thus the boundary conditions in equation (3) are correctly set, provided that the scalar functions $\phi_{0}, \phi_{L}$ are properly chosen.

\section{Discrete BLOOD FLOW MODEL}

In this section, we give the discretization of the continuous problem (11).

In the rest of this article, the upper-scripts will be devoted to time steps numbers, whereas the lower indices will in general denote the space indices, or the component index of a vector. For instance, for a vector $v$, the $\alpha^{t h}$ component of $v$ at time $t^{n}$ and at abscissa $z_{i}$ will be denoted $\left(v_{\alpha}\right)_{i}^{n}$.

\subsection{Taylor-Galerkin scheme}

We discretize our system by a second order Taylor-Galerkin scheme [1], which might be seen as the finite element counterpart of the Lax-Wendroff scheme. It has been chosen for its excellent dispersion error characteristic and its relative simplicity of implementation. See [11] for details concerning the derivation of the scheme for the blood flow model.

Let the interval $\Omega=(0, L)$ be subdivided into $N+1$ elements $e_{i+\frac{1}{2}}=\left[z_{i}, z_{i+1}\right]$, for $i=0, \ldots, N$ and $z_{i+1}=z_{i}+h_{i+\frac{1}{2}}$, with $z_{0}=0$, and $\sum_{i=0}^{N} h_{i+\frac{1}{2}}=L$, where $h_{i+\frac{1}{2}}>0$ is the local element size. Let $h>0$ be the smallest diameter of all elements $e_{i+\frac{1}{2}}, i=0, \ldots, N$. We discretize the time interval $I=\left(0, T_{f}\right)$ in the same way: let $\left(t^{n}\right), n=0, \ldots, N_{t}$, be $N_{t}+1$ instants such that $t_{0}=0<\ldots<t^{n}<t^{n+1}<\ldots<t^{N_{t}}=T_{f}$. We call $\Delta t^{n+\frac{1}{2}}=t^{n+1}-t^{n}>0$ the time step between $t^{n}$ and $t^{n+1}, n=0, \ldots, N_{t}-1$.

The space discretization is carried out using the finite element method [18]. Let $\Psi_{h}$ be the space of continuous piecewise linear finite element functions, also denoted $P_{1}$, and $\boldsymbol{\Psi}_{h}=\left[\Psi_{h}\right]^{2}$, while $\boldsymbol{\Psi}_{h, 0}=\left[\Psi_{h, 0}\right]^{2}=\left\{\boldsymbol{\psi}_{h} \in\right.$ $\boldsymbol{\Psi}_{h} \mid \boldsymbol{\psi}_{h}=\mathbf{0}$ at $z=0$ and $\left.z=L\right\}$. Let $\psi_{i}$ be the $P_{1}$ linear finite element nodal function associated to the node at $z=z_{i}, i=i=0, \ldots, N+1$. Thus one can write $\Psi_{h}=\operatorname{span}\left\{\psi_{i}, i=0, \ldots, N+1\right\}$ while $\Psi_{h, 0}=$ $\operatorname{span}\left\{\psi_{i}, i=1, \ldots, N\right\}$. We will denote a generic vector valued test function by $\boldsymbol{\psi}_{h} \in \boldsymbol{\Psi}_{h}$. The discrete continuity and momentum equations are recovered by taking test functions of the form $\boldsymbol{\psi}_{h}=\left[\psi_{h}, 0\right]^{T}$ and $\psi_{h}=\left[0, \psi_{h}\right]^{T}$, respectively, with $\psi_{h} \in \Psi_{h, 0}$.

At each time step we seek the solution $\mathbf{U}_{h} \in \boldsymbol{\Psi}_{h}$ that we may write $\mathbf{U}_{h}^{n}(z, t)=\sum_{i=0}^{N+1} \mathbf{U}_{i}^{n} \psi_{i}(z, t)$, with $\mathbf{U}_{i}^{n}=\left[A_{i}^{n}, Q_{i}^{n}\right]$ the approximation of $A$ and $Q$ at mesh node $z_{i}$.

Further, we note the $\mathbf{L}^{2}(0, L)=\mathbf{L}^{2}(\Omega)$ scalar product $(\mathbf{u}, \mathbf{v})_{\Omega}=\int_{0}^{L} \mathbf{u} \cdot \mathbf{v} d z$. 
Introducing the notations for $\mathbf{U} \in \mathbf{\Psi}_{h}, \mathbf{B}_{\mathbf{U}}=\frac{\partial \mathbf{B}}{\partial \mathbf{U}}$,

$$
\mathbf{F}_{L W}^{n}(\mathbf{U})=\mathbf{F}(\mathbf{U})-\frac{\Delta t^{n+\frac{1}{2}}}{2} \mathbf{H}(\mathbf{U}) \mathbf{B}(\mathbf{U}) \quad \text { and } \quad \mathbf{B}_{L W}^{n}(\mathbf{U})=\mathbf{B}(\mathbf{U})-\frac{\Delta t^{n+\frac{1}{2}}}{2} \mathbf{B}_{\mathbf{U}}(\mathbf{U}) \mathbf{B}(\mathbf{U})
$$

we write in equation (13) the Taylor-Galerkin discretization of the problem (11).

Given $\mathbf{U}_{h}^{0}$, for $n=0,1, \ldots, N_{t}-1$, find $\mathbf{U}_{h}^{n+1} \in \mathbf{\Psi}_{h}$ such that for all $\boldsymbol{\psi}_{h} \in \boldsymbol{\Psi}_{h, 0}$

$$
\begin{array}{ll}
\left(\mathbf{U}_{h}^{n+1}, \boldsymbol{\psi}_{h}\right)_{\Omega}= & \left(\mathbf{U}_{h}^{n}, \boldsymbol{\psi}_{h}\right)_{\Omega}+\Delta t^{n+\frac{1}{2}}\left\{\left(\mathbf{F}_{L W}^{n}\left(\mathbf{U}_{h}^{n}\right), \frac{d \boldsymbol{\psi}_{h}}{d z}\right)_{\Omega}-\left(\mathbf{B}_{L W}^{n}\left(\mathbf{U}_{h}^{n}\right), \boldsymbol{\psi}_{h}\right)_{\Omega}\right\} \\
& +\frac{\left(\Delta t^{n+\frac{1}{2}}\right)^{2}}{2}\left\{-\left(\mathbf{H}\left(\mathbf{U}_{h}^{n}\right) \frac{\partial \mathbf{F}}{\partial z}\left(\mathbf{U}_{h}^{n}\right), \frac{d \boldsymbol{\psi}_{h}}{d z}\right)_{\Omega}+\left(\mathbf{B}_{\mathbf{U}}\left(\mathbf{U}_{h}^{n}\right) \frac{\partial \mathbf{F}\left(\mathbf{U}_{h}^{n}\right)}{\partial z}, \boldsymbol{\psi}_{h}\right)_{\Omega}\right\}, \\
+ \text { B.C. } z=0 \quad \text { equation on } \mathbf{U}_{h}^{n+1}(0), \\
+ \text { B.C. } z=L & \text { equation on } \mathbf{U}_{h}^{n+1}(L) .
\end{array}
$$

In the system (13), by taking internal test functions $\boldsymbol{\psi}_{h}=\left[\psi_{i}, 0\right]^{T}$ and $\boldsymbol{\psi}_{h}=\left[0, \psi_{i}\right]^{T}$, for $i=1, \ldots N$, we obtain $\mathrm{N}$ discrete equations for continuity and momentum, respectively, for a total of $2(N+2)$ unknowns $\left(A_{i}\right.$ and $Q_{i}$ for $\left.i=0, \ldots, N+1\right)$. Thus boundary and compatibility conditions have to provide four additional scalar relations, see next section.

To fully discretize the equation in (13), we need to determine how the non-linear terms are computed and which numerical integration is performed. We choose to approximate the vectors $\mathbf{F}$ and $\mathbf{B}$ depending on $\mathbf{U}_{h}^{n}$ in (13) in the space $P_{1}$, and the matrices $\mathbf{H}$ and $\mathbf{B}_{\mathbf{U}}$ in the space of element-wise constant functions $P_{0, h}:=\left\{v_{h} \in L^{2}(\Omega)\left|v_{h}\right|_{e_{i+\frac{1}{2}}}=\mathrm{cst}, i=0, \ldots, N\right\}$. With this hypothesis, the integrations in (13) can easily be made exactly.

Thus for a vectorial function $v: \Psi_{h} \rightarrow \mathbb{R}^{2}, \mathbf{U} \mapsto v(\mathbf{U})=\left[v_{1}, v_{2}\right]^{\top}$, we define

$$
v_{h}(\mathbf{U})=\left[\sum_{i=0}^{N+1} v_{1}\left(\mathbf{U}_{i}\right) \psi_{i}, \sum_{i=0}^{N+1} v_{2}\left(\mathbf{U}_{i}\right) \psi_{i}\right]^{\top} \in \mathbf{\Psi}_{h},
$$

and for a matrix $M: \Psi_{h} \rightarrow \mathbb{R}^{2 \times 2}, \mathbf{U} \mapsto M(\mathbf{U})=\left(M_{\alpha \beta}\right)_{\alpha, \beta=1,2}$, we define

$$
M_{h}(\mathbf{U})=\left(M_{h, \alpha \beta}(\mathbf{U})\right)_{\alpha, \beta=1,2}, \quad M_{h, \alpha \beta}(\mathbf{U})=\sum_{i=0}^{N} \tilde{M}_{h, \alpha \beta, i+\frac{1}{2}} 1_{i+\frac{1}{2}} \in P_{0, h}
$$

with the mean value $\tilde{M}_{h, \alpha \beta, i+\frac{1}{2}}=\frac{1}{2}\left(M_{\alpha \beta}\left(\mathbf{U}_{i}\right)+M_{\alpha \beta}\left(\mathbf{U}_{i+1}\right)\right)$ over the element $e_{i+\frac{1}{2}}=\left[z_{i}, z_{i+1}\right]$, and the characteristic function $1_{i+\frac{1}{2}}$ of the interval $\left[z_{i}, z_{i+1}\right]$, for $i=0, \ldots, N$.

With these notations, we can write the fully discretized Taylor-Galerkin scheme (13). Using the notation

$$
\mathbf{F}_{h, L W}^{n}(\mathbf{U})=\mathbf{F}_{h}(\mathbf{U})-\frac{\Delta t^{n+\frac{1}{2}}}{2} \mathbf{H}_{h}(\mathbf{U}) \mathbf{B}_{h}(\mathbf{U}) \quad \text { and } \quad \mathbf{B}_{h, L W}(\mathbf{U})=\mathbf{B}_{h}(\mathbf{U})-\frac{\Delta t^{n+\frac{1}{2}}}{2}\left(\mathbf{B}_{\mathbf{U}}\right)_{h}(\mathbf{U}) \mathbf{B}_{h}(\mathbf{U})
$$

and introducing the operator for $\mathbf{U}, \mathbf{W} \in \mathbf{\Psi}_{h}$

$$
\begin{aligned}
a_{h}^{n}(\mathbf{U}, \mathbf{W} ; \mathbf{p})= & \Delta t^{n+\frac{1}{2}}\left\{\left(\mathbf{F}_{h, L W}^{n}(\mathbf{U}), \frac{d \mathbf{W}}{d z}\right)_{\Omega}-\left(\mathbf{B}_{h, L W}^{n}(\mathbf{U}), \mathbf{W}\right)_{\Omega}\right\} \\
& +\frac{\left(\Delta t^{n+\frac{1}{2}}\right)^{2}}{2}\left\{-\left(\mathbf{H}_{h}(\mathbf{U}) \frac{\partial \mathbf{F}_{h}}{\partial z}(\mathbf{U}), \frac{d \mathbf{W}}{d z}\right)_{\Omega}+\left(\left(\mathbf{B}_{\mathbf{U}}\right)_{h}(\mathbf{U}) \frac{\partial \mathbf{F}_{h}}{\partial z}(\mathbf{U}), \mathbf{W}\right)_{\Omega}\right\}
\end{aligned}
$$


the fully discretized scheme (13) yields:

Given $\mathbf{U}_{h}^{0} \in \mathbf{\Psi}_{h}$, find $\mathbf{U}_{h}:=\left(\mathbf{U}_{h}^{n}\right)_{n=1, \ldots, N_{t}} \in\left(\mathbf{\Psi}_{h}\right)^{N_{t}}$ such that for $n=0,1, \ldots, N_{t}-1$

$$
\begin{aligned}
\left(\mathbf{U}_{h}^{n+1}, \boldsymbol{\psi}_{h}\right)_{\Omega}= & \left(\mathbf{U}_{h}^{n}, \boldsymbol{\psi}_{h}\right)_{\Omega}+a_{h}^{n}\left(\mathbf{U}_{h}^{n}, \boldsymbol{\psi}_{h} ; \mathbf{p}\right) \forall \boldsymbol{\psi}_{h} \in \boldsymbol{\Psi}_{h, 0}, \\
& \text { B.C. on } \mathbf{U}_{h, 0}^{n+1}, \\
& \text { B.C. on } \mathbf{U}_{h, N+1}^{n+1} .
\end{aligned}
$$

In the discrete approximate problem (16), all the integrals can be computed exactly as they involve only the products of two functions belonging either to $P_{1}$ or to $P_{0}$.

We need to add boundary conditions and compatibility conditions at $z=0$ and $z=L$ to close the system.

\subsection{Boundary conditions}

In this section and in the next sections 2.3 and 2.4, we will omit the subscript $h$ in the variable names, for the sake of simplicity.

As noticed in Remark 1.1, the hyperbolic problem (11) is well posed under sub-critical flow hypothesis when one imposes as boundary conditions one scalar equation at each side of the tube. These conditions can be the prescribed incoming characteristics, the prescribed pressure or the prescribed flux, for instance. It is not possible to impose exactly at the same boundary both the flux and the pressure, see [11].

We decide to give a flux at the inlet and a pressure at the outlet:

$$
\begin{aligned}
Q_{0}^{n+1} & =\phi_{0}\left(q_{0}\left(t^{n+1}\right), \mathbf{p}\right)=q_{0}\left(t^{n+1}\right) \\
A_{N+1}^{n+1} & =\phi_{L}\left(p_{L}\left(t^{n+1}\right), \mathbf{p}\right)
\end{aligned}
$$

where $q_{0}$ is the flux and $p_{L}$ is the pressure to be imposed at the boundaries, whereas $\phi_{i}, i=0, L$ are given functions. The function $\phi_{0}$ is in this case the identity, and $\phi_{L}$ transforms the given pressure $p_{L}$ at the outlet into the conservative unknown $A$. With the pressure law $(4)$, it becomes $A=\phi_{L}\left(p_{L}, \mathbf{p}\right)=A_{0}\left(1+\frac{p_{L}}{\beta_{0}}\right)^{1 / \beta_{1}}$.

\subsection{Compatibility conditions}

To close the discrete system we need two more conditions on the boundaries. These conditions are a numerical artefact that is linked to the type of scheme we adopted. They are called compatibility conditions and are chosen to be non-reflecting conditions at each side of the tube. We assume that these conditions are treated explicitly and linearly, imposing some conditions on the pseudo-characteristic variables, [19]. Thus they can take the following form:

$$
\begin{aligned}
& \mathbf{l}_{2}^{\top}\left(\mathbf{U}^{n}\right) \mathbf{U}_{0}^{n+1}-T_{2}\left(\mathbf{U}^{n}\right)=0 \quad \text { at } z=0, \\
& \mathbf{l}_{1}^{\top}\left(\mathbf{U}^{n}\right) \mathbf{U}_{N+1}^{n+1}-T_{1}\left(\mathbf{U}^{n}\right)=0 \quad \text { at } z=L,
\end{aligned}
$$

where $\mathbf{l}_{i}\left(\mathbf{U}^{n}\right) \in \mathbb{R}^{2}, i=1,2$ are the two left eigenvectors depending on $\mathbf{U}^{n}$ associated to the matrix $\mathbf{H}$ defined in equation (12), and $T_{1}$ and $T_{2}$ are some scalar functions depending on $\mathbf{U}^{n}$.

\subsection{Fully discretized problem}

Gathering the boundary and compatibility conditions (17) and (18) and reintroducing the parameters p, we obtain

with at $z=z_{0}=0$

$$
\begin{aligned}
\Theta_{0}\left(\mathbf{U}^{n}, \mathbf{p}\right) \mathbf{U}_{0}^{n+1}-\mathbf{T}_{0}\left(\mathbf{U}^{n}, \mathbf{p}\right) & =0 \\
\Theta_{L}\left(\mathbf{U}^{n}, \mathbf{p}\right) \mathbf{U}_{N+1}^{n+1}-\mathbf{T}_{L}\left(\mathbf{U}^{n}, \mathbf{p}\right) & =0
\end{aligned}
$$

$$
\Theta_{0}\left(\mathbf{U}^{n}, \mathbf{p}\right)=\left[\begin{array}{c}
\Theta_{0,1}^{\top} \\
\Theta_{0,2}^{\top}\left(\mathbf{U}^{n}, \mathbf{p}\right)
\end{array}\right]=\left[\begin{array}{cc}
0 & 1 \\
\mathbf{l}_{2}^{\top}\left(\mathbf{U}^{n}, \mathbf{p}\right)
\end{array}\right], \quad \mathbf{T}_{0}\left(\mathbf{U}^{n}, \mathbf{p}\right)=\left[\begin{array}{c}
q_{0}\left(t^{n+1}\right) \\
T_{2}\left(\mathbf{U}^{n}, \mathbf{p}\right)
\end{array}\right],
$$


and at $z=z_{N+1}=L$

$$
\Theta_{L}\left(\mathbf{U}^{n}, \mathbf{p}\right)=\left[\begin{array}{c}
\Theta_{L, 1}^{\top}\left(\mathbf{U}^{n}, \mathbf{p}\right) \\
\Theta_{L, 2}^{\top}
\end{array}\right]=\left[\begin{array}{c}
\mathbf{l}_{1}^{\top}\left(\mathbf{U}^{n}, \mathbf{p}\right) \\
1 \\
0
\end{array}\right], \quad \mathbf{T}_{L}\left(\mathbf{U}^{n}, \mathbf{p}\right)=\left[\begin{array}{c}
T_{1}\left(\mathbf{U}^{n}, \mathbf{p}\right) \\
\phi_{L}\left(p_{L}\left(t^{n+1}\right), \mathbf{p}\right)
\end{array}\right]
$$

Finally, reintroducing the subscript $h$, adding the boundary and compatibility conditions (19) to the completely discretized scheme (16), the problem to be solved reads:

Given $\mathbf{U}_{h}^{0} \in \mathbf{\Psi}_{h}$, find $\mathbf{U}_{h}:=\left(\mathbf{U}_{h}^{n}\right)_{n=1, \ldots, N_{t}} \in\left(\mathbf{\Psi}_{h}\right)^{N_{t}}$ such that for $n=0,1, \ldots, N_{t}-1$

$$
\begin{array}{ll}
\left(\mathbf{U}_{h}^{n+1}, \boldsymbol{\psi}_{h}\right)_{\Omega} & =\left(\mathbf{U}_{h}^{n}, \boldsymbol{\psi}_{h}\right)_{\Omega}+a_{h}^{n}\left(\mathbf{U}_{h}^{n}, \boldsymbol{\psi}_{h} ; \mathbf{p}\right) \quad \forall \boldsymbol{\psi}_{h} \in \boldsymbol{\Psi}_{h, 0}, \\
\Theta_{0}\left(\mathbf{U}_{h}^{n}, \mathbf{p}\right) \mathbf{U}_{h, 0}^{n+1} & =\mathbf{T}_{0}\left(\mathbf{U}_{h}^{n}, \mathbf{p}\right) \\
\Theta_{L}\left(\mathbf{U}_{h}^{n}, \mathbf{p}\right) \mathbf{U}_{h, N+1}^{n+1} & =\mathbf{T}_{L}\left(\mathbf{U}_{h}^{n}, \mathbf{p}\right)
\end{array}
$$

Remark 2.1. The problem (11) is a 1-d nonlinear hyperbolic problem, but the Taylor-Galerkin discretization (20) contains (second order) parabolic terms. Thus, one needs to impose not only the boundary conditions (17) that are natural for a hyperbolic problem, but also the compatibility conditions (18). Therefore at each time step, before computing the solution at internal nodes, one has to compute the boundary values $\mathbf{U}_{h, 0}^{n+1}$ and $\mathbf{U}_{h, N+1}^{n+1}$, by solving the two $(2 \times 2)$ linear systems $(19)$.

\section{Gradient of the Discrete problem}

We present in this section the gradient of the discrete problem (20) using the adjoint approach. We recall that we used and implemented the rule (advocated in [3], [4], [5]): disretize first, differentiate after. In Section 3.1, some notations are defined in order to present briefly in Section 3.2 the adjoint state approach, and to compute analytically the gradient in Section 3.3.

\subsection{Forward operators}

\subsubsection{State equation}

The equation (20) defines a state equation that relates the parameters $\mathbf{p}$ to the state variables $\mathbf{U}_{h}$

$$
E\left(\mathbf{p}, \mathbf{U}_{h}\right)=0
$$

where the parameters are

$$
\mathbf{p}=\left(\alpha, \beta_{0}, \beta_{1}, K_{r}, A_{0}, q_{0}, q_{L}\right)^{\top} \in \mathbb{R}^{\mathcal{P}},
$$

with the dimension $\mathcal{P}>0$ of the parameter space to be defined further, and the state variable are

$$
\mathbf{U}_{h}=\left(\mathbf{U}_{h}^{n}\right)_{n=1, \ldots, N_{t}}=\left(\left[A_{i}^{n}, Q_{i}^{n}\right]^{\top}\right)_{i=0, \ldots, N+1 ; n=1, \ldots, N_{t}} \in\left(\Psi_{h}\right)^{N_{t}}=\mathbb{R}^{\mathcal{N}}, \mathcal{N}=2 N_{t}(N+2) .
$$

In equation (23), we have assimilated the finite functional space $\left(\boldsymbol{\Psi}_{h}\right)^{N_{t}}$ and the space $\mathbb{R}^{\mathcal{N}}$. It will also be done for $\boldsymbol{\Psi}_{h}$ and $\mathbb{R}^{2(N+2)}$. In the rest of the article, for simplicity but with an abuse of notation, we may use the same notation $\mathbf{W}_{h}^{n}$ for an element of $\boldsymbol{\Psi}_{h}$ or of $\mathbb{R}^{2(N+2)}$.

As there exists a unique solution $\mathbf{U}_{\mathbf{p}, h}$ to the problem (21) for a given set of parameters $\mathbf{p}$, see [11], we can define the direct application

$$
\begin{aligned}
\varphi: \mathbb{R}^{\mathcal{P}} & \longrightarrow \mathbb{R}^{\mathcal{N}}, \\
\mathbf{p} & \longmapsto \varphi(\mathbf{p})=\mathbf{U}_{\mathbf{p}, h},
\end{aligned}
$$

The parameters $\mathbf{p}$ in (22) are supposed to be constant with time and are to be estimated. The issue of the parameterization will be addressed in the section 3.1.5. 


\subsubsection{Measurement operator}

Let $M$ be a measurement (or observation) operator

$$
\begin{aligned}
M: \mathbb{R}^{\mathcal{N}} & \longrightarrow \mathbb{R}^{\mathcal{M}}, \\
\mathbf{U} & \longmapsto M(\mathbf{U})=\mathbf{V},
\end{aligned}
$$

with $\mathcal{M}>0$ the dimension of the measurement space. Different measurement operators can be devised, linear or nonlinear, according to the type of data that are provided.

For instance, an experiment could provide the flux and area of a vessel at two different points $\zeta_{0}<\zeta_{1}, \zeta_{1}-\zeta_{0}=$ $L$, at given $\left(\mathcal{V}_{t}+1\right)$ instants $\theta_{0}=0<\ldots<\theta^{\nu}<\theta^{\nu+1}<\ldots<\theta^{\mathcal{V}_{t}}=T_{f}$. It is then reasonable to perform the 1 -d simulation between these two points, and therefore take $z_{0}=\zeta_{0}$ and $z_{N+1}=\zeta_{1}$. One can also assume for simplicity that the temporal discretization is chosen such that the instants $\left(\theta^{\nu}\right)_{\nu=0, \ldots, \mathcal{V}_{t}}$ constitutes a subsequence of $\left(t^{n}\right)_{n=0, \ldots, N_{t}}$. Therefore, let $\phi_{t}:\left\{0,1, \ldots, \mathcal{V}_{t}\right\} \rightarrow\left\{0,1, \ldots, N_{t}\right\}$ be the increasing function such that $t^{\phi_{t}(\nu)}=\theta^{\nu}$, with $\phi_{t}(0)=0$ and $\phi_{t}\left(\mathcal{V}_{t}\right)=N_{t}$. With these hypotheses, the measurement operator is simply a weighted sampling of the direct solution $\mathbf{U}_{\mathbf{p}, h}$. It is linear and can be expressed

$$
M(\mathbf{U})=\left(\left[w_{i, \nu}^{A} A_{i}^{\phi_{t}(\nu)}, w_{i, \nu}^{Q} Q_{i}^{\phi_{t}(\nu)}\right]^{\top}\right)_{i=0 \text { and } N+1 ; \nu=0, \ldots, \mathcal{V}_{t}} \quad \text { and } \quad \mathcal{M}=2 \times\left(\mathcal{V}_{t}+1\right) \times 2,
$$

where the coefficients $w_{i, \nu}^{A}$ and $w_{i, \nu}^{Q}$ are positive weights attributed to each measure, representing the "degree of confidence", i.e. the inverse of the incertainty, one has in this measure.

When the data come from a numerical 3-d fluid structure computation, one can exploit the numerical results on $\left(\mathcal{V}_{z}+1\right)$ internal sections $\left(\zeta_{j}\right)_{j=0, \ldots, \mathcal{V}_{z}}$ of the 3 -d domain: $\mathcal{V}_{z}=1,2$ or more. In this case, apply the same technique for the spatial discretization as the one shown above for the temporal sampling, and introduce the increasing indexation function $\phi_{z}$ in the same manner as $\phi_{t}$. The measurement operator becomes this time

$$
M(\mathbf{U})=\left(\left[w_{j, \nu}^{A} A_{\phi_{z}(j)}^{\phi_{t}(\nu)}, w_{j, \nu}^{Q} Q_{\phi_{z}(j)}^{\phi_{t}(\nu)}\right]^{\top}\right)_{j=0, \ldots, \mathcal{V}_{z} ; \nu=0, \ldots, \mathcal{V}_{t}} \quad \text { and } \quad \mathcal{M}=2\left(\mathcal{V}_{t}+1\right)\left(\mathcal{V}_{z}+1\right)
$$

with the positive weights $w_{j, \nu}^{A}$ and $w_{j, \nu}^{Q}, j=0, \ldots, \mathcal{V}_{z} ; \nu=0, \ldots, \mathcal{V}_{t}$.

\subsubsection{Forward function}

Let $F=M \circ \varphi$ be the forward function, from the parameter space to the measure space

$$
\begin{aligned}
F: \mathbb{R}^{\mathcal{P}} & \longrightarrow \mathbb{R}^{\mathcal{M}}, \\
\mathbf{p} & \longmapsto F(\mathbf{p})=\mathbf{V}_{\mathbf{p}, h}=M\left(\mathbf{U}_{\mathbf{p}, h}\right)=M(\varphi(\mathbf{p})) .
\end{aligned}
$$

\subsubsection{Cost function}

The resolution of the inverse problem consists in minimizing a cost function that computes the least squares error between the measured data and the numerical results computed with the 1-d model. Thus the inverse problem amounts to an optimization problem. To solve it, it is necessary to define the cost function to minimize, that depends on the type of problem under consideration. Then one must choose an adequate technique to perform the minimization of this cost function.

We assume that the measurement operator is defined by (26). Consider a given data vector $Z \in \mathbb{R}^{\mathcal{M}}$ and we call $Z_{w} \in \mathbb{R}^{\mathcal{M}}$ this vector multiplied by the positive weights introduced in (26),

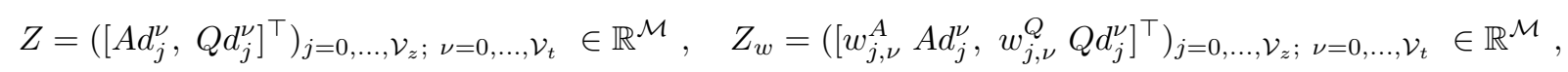

where $A d_{j}^{\nu}$, and respectively $Q d_{j}^{\nu}$, represent the data area, respectively the data flux, given at space index $j$ and time index $\nu$. 
Let $J$ be the cost function

$$
\begin{aligned}
J: \mathbb{R}^{\mathcal{P}} & \longrightarrow \mathbb{R} \\
\mathbf{p} & \longmapsto J(\mathbf{p})=\frac{1}{2}\left\|Z_{w}-M\left(\mathbf{U}_{\mathbf{p}, h}\right)\right\|_{\mathbb{R}^{\mathcal{M}}}^{2}
\end{aligned}
$$

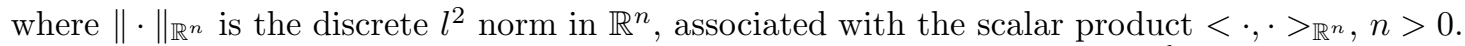

The cost function can be expressed easily, with $\mathbf{U}_{\mathbf{p}, h}=\left(\left[A_{i}^{n}, Q_{i}^{n}\right]^{\top}\right) \in \mathbb{R}^{\mathcal{N}}$,

$$
J(\mathbf{p})=\frac{1}{2} \sum_{\nu=0}^{\mathcal{V}_{t}} \sum_{j=0}^{\mathcal{V}_{z}}\left\{\left(w_{j, \nu}^{A}\right)^{2}\left(A d_{j}^{\nu}-A_{\phi_{z}(j)}^{\phi_{t}(\nu)}\right)^{2}+\left(w_{j, \nu}^{Q}\right)^{2}\left(Q d_{j}^{\nu}-Q_{\phi_{z}(j)}^{\phi_{t}(\nu)}\right)^{2}\right\}
$$

\subsubsection{Parameterization}

It is important to define the parameterization properly, see [6], and a sensitivity analysis can provide some information on this matter. In this section, we consider a general parameterization. The parameters are only supposed to satisfy some constraints determined by some physiological considerations, such that the parameters p belong to a subset $C$ of $\mathbb{R}^{\mathcal{P}}$.

\subsection{Optimization: adjoint state approach}

In order to perform correctly the optimization of the cost function (28) with a descent method, one needs to compute the gradient of the cost function in a fast and accurate way. It is known that the finite difference method is not efficient, see [4,13]. One could rely on a direct computation of the jacobian of the forward map $F$, with a cost that is proportional to the number $\mathcal{P}$ of parameters. We decided to compute the gradient using an adjoint state approach that we present briefly here. As in our numerical experiments, see section 4, the number of parameters to identify is very low, we could have fruitfully taken the former approach. However, we preferred the adjoint method, that allows to identify, in a second step, more parameters, with a cost that remains independent of the dimension of the parameter space.

To compute the gradient of a function $G=G(\mathbf{p}, \mathbf{V})$ which is an explicit function of the parameter vector $\mathbf{p}$ and the output vector $\mathbf{V}=F(\mathbf{p})$, we introduce the Lagrangian

$$
\mathcal{L}(\mathbf{p}, \mathbf{U}, \lambda)=G(\mathbf{p}, M(\mathbf{U}))+<E(\mathbf{p}, \mathbf{U}), \lambda>_{\mathbb{R}^{\mathcal{N}}},
$$

where the Lagrange multiplier $\lambda \in \mathbb{R}^{\mathcal{N}}$ is the adjoint variable of $\mathbf{U}$.

With these notations, we can state the following proposition, (see [3]):

Proposition 3.1. Let (21) be a state equation and (25) an observation operator. Let $G(\mathbf{p}, \mathbf{V})$ and $F(\mathbf{p})$, given by (27), two regular enough functions. Let $\mathcal{L}(\mathbf{p}, \mathbf{U}, \lambda)$ be a Lagrangian defined by (30) associated with the equation (21).

Then $\mathbf{p} \in C \subset \mathbb{R}^{\mathcal{P}} \mapsto G(\mathbf{p}, F(\mathbf{p})) \in \mathbb{R}$ is differentiable, and its gradient $\nabla G$ is given by the gradient equation:

$$
<\nabla G, \delta \mathbf{p}>_{\mathbb{R}^{\mathcal{P}}}=\frac{\partial \mathcal{L}}{\partial \mathbf{p}}\left(\mathbf{p}, \mathbf{U}_{\mathbf{p}}, \lambda_{\mathbf{p}}\right) \delta \mathbf{p} \quad \forall \delta \mathbf{p} \in \mathbb{R}^{\mathcal{P}}
$$

where

$* \mathbf{U}_{\mathbf{p}} \in \mathbb{R}^{\mathcal{N}}$ is the solution of the direct equation

$$
E(\mathbf{p}, \mathbf{U})=0
$$

$* \lambda_{\mathbf{p}} \in \mathbb{R}^{\mathcal{N}}$ is the solution of the adjoint equation

$$
\frac{\partial \mathcal{L}}{\partial \mathbf{U}}\left(\mathbf{p}, \mathbf{U}_{\mathbf{p}}, \lambda\right) \delta \mathbf{U}=0 \quad \forall \delta \mathbf{U} \in \delta \mathbb{R}^{\mathcal{N}}
$$


In this context, $\mathbf{U}_{\mathbf{p}}$ is called the direct state, and $\lambda_{\mathbf{p}}$ the adjoint state.

From the formula (33), the adjoint equation yields:

$$
\left\{\frac{\partial E}{\partial \mathbf{U}}(\mathbf{p}, \mathbf{U})\right\}^{\top} \lambda+M^{\prime}(\mathbf{U})^{\top} \nabla_{\mathbf{V}} G(\mathbf{p}, \mathbf{V})=0
$$

This presentation has the advantage of synthetizing the different applications according to the choice on function $G(\mathbf{p}, \mathbf{V})$ :

- if $G(\mathbf{p}, \mathbf{V})=<\mathbf{V}, e_{i}>_{\mathbb{R}^{\mathcal{M}}}$ where $e_{i}$ is a basis vector of the measure space, then:

$$
\nabla G=F^{\prime}(\mathbf{p})^{\top} e_{i},
$$

and the adjoint approach enables a line-wise computation of the jacobian of the function $F(\mathbf{p})$.

- if $G(\mathbf{p}, \mathbf{V})=<\mathbf{V}, g_{v}>_{\mathbb{R}} \mathcal{M}$ where $g_{v}$ is a given vector in $\mathbb{R}^{\mathcal{M}}$, then:

$$
\nabla G=F^{\prime}(\mathbf{p})^{\top} g_{v}
$$

- if $G(\mathbf{p}, \mathbf{V})=\frac{1}{2}\left\|Z_{w}-\mathbf{V}\right\|^{2}$, i.e. is equal to the cost function $J(\mathbf{p})$, then:

$$
\nabla G=\nabla J
$$

\subsection{Gradient of the discrete problem}

We compute in this section the gradient of the discrete problem (20) using the adjoint approach. First, we define the Lagrangian in section 3.3.1, then we differentiate the Lagrangian with respect to the state variable to obtain the adjoint problem in section 3.3.2. Finally, the gradient is computed by differentiation with respect to the parameters in section 3.3.3.

\subsubsection{Lagrangian of the discrete problem}

We define the Lagrangian of our particular discrete 1-d blood flow model (20). Two sets of Lagrange multipliers are introduced. The first ones are associated with the boundary conditions (19) at $z=0$ and $z=L$ :

$$
\mu_{z i, h}=\left(\mu_{z i, h}^{n}\right)_{n=1, \ldots, N_{t}} \in\left(\mathbb{R}^{2}\right)^{N_{t}}, \quad i=0, L,
$$

and the second ones are associated with the equations on the internal nodes (16) and live in the space of the test functions:

$$
\lambda_{h}=\left(\lambda_{h}^{n}\right)_{n=1, \ldots, N_{t}} \in\left(\Psi_{h, 0}\right)^{N_{t}}=\left(\mathbb{R}^{2}\right)^{N_{t} \times N} .
$$

The Lagrangian associated with the forward discrete problem (20) and to the generic function $G=G(\mathbf{p}, \mathbf{V})$ is hence:

$$
\begin{aligned}
\mathcal{L}\left(\mathbf{p}, \mathbf{U}, \lambda_{h}, \mu_{z 0, h}, \mu_{z L, h}\right) & =G\left(\mathbf{p}, M\left(\mathbf{U}_{h}\right)\right) \\
& +\sum_{n=0}^{N_{t}-1}\left[\left(\mathbf{U}_{h}^{n+1}, \lambda_{h}^{n+1}\right)_{\Omega}-\left(\mathbf{U}_{h}^{n}, \lambda_{h}^{n+1}\right)_{\Omega}-a_{h}^{n}\left(\mathbf{U}_{h}^{n}, \lambda_{h}^{n+1} ; \mathbf{p}\right)\right] \\
& +\sum_{n=0}^{N_{t}-1} \sum_{i=0, L}\left[\Theta_{i}\left(\mathbf{U}_{h}^{n}, \mathbf{p}\right) \mathbf{U}_{h, i}^{n+1}-\mathbf{T}_{i}\left(\mathbf{U}_{h}^{n}, \mathbf{p}\right)\right] \cdot \mu_{z i, h}^{n+1} .
\end{aligned}
$$

where $\mathbf{U}_{h, L}=\mathbf{U}_{h, N+1}=\mathbf{U}_{h}(t, L)$. 


\subsubsection{Discrete adjoint problem}

We differentiate the Lagrangian (37) with respect to the state $\mathbf{U}_{h}$, remarking that $\delta \mathbf{U}_{h}^{0}=0$, and omitting for the sake of conciseness the dependency to the parameters $\mathbf{p}$, to obtain

$$
\begin{gathered}
\frac{\partial \mathcal{L}}{\partial \mathbf{U}_{h}}\left(\mathbf{p}_{h}, \mathbf{U}_{h}, \lambda_{h}, \mu_{z 0, h}, \mu_{z L, h}\right) \delta \mathbf{U}_{h}=\left(\frac{\partial M}{\partial \mathbf{U}_{h}}\left(\mathbf{U}_{h}\right)\right)^{\top}\left(\frac{\partial G}{\partial \mathbf{V}}\right)^{\top} \cdot \delta \mathbf{U}_{h} \\
+\sum_{n=1}^{N_{t}-1}\left[\left(\delta \mathbf{U}_{h}^{n+1}, \lambda_{h}^{n+1}\right)_{\Omega}-\left(\delta \mathbf{U}_{h}^{n}, \lambda_{h}^{n+1}\right)_{\Omega}-\frac{\partial a_{h}^{n}}{\partial \mathbf{U}_{h}}\left(\mathbf{U}_{h}^{n}, \delta \mathbf{U}_{h}^{n}, \lambda_{h}^{n+1}\right)\right] \\
+\sum_{n=1}^{N_{t}-1} \sum_{i=0, L}\left[\Theta_{i}\left(\mathbf{U}_{h}^{n}\right) \delta \mathbf{U}_{h, i}^{n+1}+\frac{\partial \Theta_{i}}{\partial \mathbf{U}_{h}}\left(\mathbf{U}_{h}^{n}\right)\left(\delta \mathbf{U}_{h}^{n}, \mathbf{U}_{h, i}^{n+1}\right)-\frac{\partial \mathbf{T}_{i}}{\partial \mathbf{U}_{h}}\left(\mathbf{U}_{h}^{n}\right) \delta \mathbf{U}_{h}^{n}\right] \cdot \mu_{z i, h}^{n+1} \\
+\left(\delta \mathbf{U}_{h}^{1}, \lambda_{h}^{1}\right)_{\Omega}+\sum_{i=0, L}\left[\Theta_{i}\left(\mathbf{U}_{h}^{0}\right) \delta \mathbf{U}_{h, i}^{1}\right] \cdot \mu_{z i, h}^{1} \\
=0 \quad \forall \delta \mathbf{U}_{h} \in\left(\mathbf{\Psi}_{h}\right)^{N_{t}} .
\end{gathered}
$$

In equation (38), we formulated explicitely the dependency of the term $\frac{\partial a_{h}^{n}}{\partial \mathbf{U}_{h}}$ with respect to $\mathbf{U}_{h}$ and $\delta \mathbf{U}_{h}$, that derives from the nonlinearity of the operator $a_{h}^{n}$. A discrete integration by parts in time to order the terms in function of $\delta \mathbf{U}_{h}^{n}$ yields

$$
\begin{aligned}
& \frac{\partial \mathcal{L}}{\partial \mathbf{U}_{h}}\left(\mathbf{p}_{h}, \mathbf{U}_{h}, \lambda_{h}, \mu_{z 0, h}, \mu_{z L, h}\right) \delta \mathbf{U}_{h}==\left[\nabla_{\mathbf{U}_{h}} M\left(\mathbf{U}_{h}\right) \nabla_{\mathbf{V}} G\right] \cdot \delta \mathbf{U}_{h} \\
&+\left(\delta \mathbf{U}_{h}^{N_{t}}, \lambda_{h}^{N_{t}}\right)_{\Omega}+\sum_{i=0, L}\left[\Theta_{i}\left(\mathbf{U}_{h}^{N_{t}-1}\right) \delta \mathbf{U}_{h, i}^{N_{t}}\right] \cdot \mu_{z i, h}^{N_{t}} \\
&+\sum_{n=1}^{N_{t}-1}\left[\left(\delta \mathbf{U}_{h}^{n}, \lambda_{h}^{n}\right)_{\Omega}-\left(\delta \mathbf{U}_{h}^{n}, \lambda_{h}^{n+1}\right)_{\Omega}-\frac{\partial a_{h}^{n}}{\partial \mathbf{U}_{h}}\left(\mathbf{U}_{h}, \delta \mathbf{U}_{h}^{n}, \lambda_{h}^{n+1}\right)\right] \\
&+\sum_{n=1}^{N_{t}-1} \sum_{i=0, L}\left[\Theta_{i}\left(\mathbf{U}_{h}^{n-1}\right) \delta \mathbf{U}_{h, i}^{n}\right] \cdot \mu_{z i, h}^{n} \\
&+\sum_{n=1}^{N_{t}-1} \sum_{i=0, L}\left[\frac{\partial \Theta_{i}}{\partial \mathbf{U}_{h}}\left(\mathbf{U}_{h}^{n}\right)\left(\delta \mathbf{U}_{h}^{n}, \mathbf{U}_{h, i}^{n+1}\right)-\frac{\partial \mathbf{T}_{i}}{\partial \mathbf{U}_{h}}\left(\mathbf{U}_{h}^{n}\right) \delta \mathbf{U}_{h}^{n}\right] \cdot \mu_{z i, h}^{n+1} \\
&=0 \quad \forall \delta \mathbf{U}_{h} \in\left(\mathbf{\Psi}_{h}\right)^{N_{t}} .
\end{aligned}
$$

First, taking care of the final time $t^{N_{t}}$, i.e. $\delta \mathbf{U}_{h}=\delta \mathbf{U}_{h}^{N_{t}}$, we take successively internal test functions $\delta \mathbf{U}_{h}^{N_{t}}=\boldsymbol{\psi}_{h} \in \boldsymbol{\Psi}_{h, 0}$ and boundary test functions $\delta \mathbf{U}_{h}^{N_{t}}=\boldsymbol{\psi}_{h, i} \in \boldsymbol{\Psi}_{h}, i=0, L$, where $\boldsymbol{\psi}_{h, 0}(0)=\boldsymbol{\psi}_{h, L}(L)=1$. Thus we have to solve the two following problems at time $t^{N_{t}}$ :

Find $\lambda_{h}^{N_{t}} \in \boldsymbol{\Psi}_{h, 0}$ such that, for all $\boldsymbol{\psi}_{h} \in \boldsymbol{\Psi}_{h, 0}$

$$
\left(\lambda_{h}^{N_{t}}, \boldsymbol{\psi}_{h}\right)_{\Omega}=-\left[\nabla_{\mathbf{U}_{h}} M\left(\mathbf{U}_{h}^{N_{t}}\right) \nabla_{\mathbf{V}} G\right] \cdot \boldsymbol{\psi}_{h}
$$

and then find $\mu_{z i, h}^{N_{t}} \in \mathbb{R}^{2}, i=0, L$ such that

$$
\Theta_{i}^{\top}\left(\mathbf{U}_{h}^{N_{t}-1}\right) \mu_{z i, h}^{N_{t}}=-\left[\nabla_{\mathbf{U}_{h}} M\left(\mathbf{U}_{h}^{N_{t}}\right) \nabla_{\mathbf{V}} G\right] \cdot \boldsymbol{\psi}_{h, i}-\left(\lambda_{h}^{N_{t}}, \boldsymbol{\psi}_{h, i}\right)_{\Omega}
$$

Next, we take care successively of time steps $n, n=N_{t}-1, N_{t}-2, \ldots, 1$. We use as test functions $\delta \mathbf{U}_{h}^{n}=$ $\boldsymbol{\psi}_{h} \in \boldsymbol{\Psi}_{h, 0}$ and $\delta \mathbf{U}_{h}^{n}=\boldsymbol{\psi}_{h, i}, i=0, L$. Thus we have to solve the two following problems at time $t^{n}$ : 
Find $\lambda_{h}^{n} \in \boldsymbol{\Psi}_{h, 0}$ such that, for all $\boldsymbol{\psi}_{h} \in \boldsymbol{\Psi}_{h, 0}$

$$
\begin{aligned}
\left(\lambda_{h}^{n}, \boldsymbol{\psi}_{h}\right)_{\Omega}= & -\left[\nabla_{\mathbf{U}_{h}} M\left(\mathbf{U}_{h}^{n}\right) \nabla_{\mathbf{V}} G\right] \cdot \boldsymbol{\psi}_{h}+\left(\lambda_{h}^{n+1}, \boldsymbol{\psi}_{h}\right)_{\Omega}+\frac{\partial a_{h}^{n}}{\partial \mathbf{U}_{h}}\left(\mathbf{U}_{h}^{n}, \boldsymbol{\psi}_{h}, \lambda_{h}^{n+1}\right) \\
& -\sum_{i=0, L}\left[\frac{\partial \Theta_{i}}{\partial \mathbf{U}_{h}}\left(\mathbf{U}_{h}^{n}\right)\left(\boldsymbol{\psi}_{h}, \mathbf{U}_{h, i}^{n+1}\right)-\frac{\partial \mathbf{T}_{i}}{\partial \mathbf{U}_{h}}\left(\mathbf{U}_{h}^{n}\right) \boldsymbol{\psi}_{h}\right] \cdot \mu_{z i, h}^{n+1},
\end{aligned}
$$

and then find $\mu_{z i, h}^{n} \in \mathbb{R}^{2}, i=0, L$ such that

$$
\begin{aligned}
\Theta_{i}^{\top}\left(\mathbf{U}_{h}^{n-1}\right) \mu_{z i, h}^{n}= & -\left[\nabla_{\mathbf{U}_{h}} M\left(\mathbf{U}_{h}^{n}\right) \nabla_{\mathbf{V}} G\right] \cdot \boldsymbol{\psi}_{h, i} \\
& -\left(\lambda_{h}^{n}, \boldsymbol{\psi}_{h, i}\right)_{\Omega}+\left(\lambda_{h}^{n+1}, \boldsymbol{\psi}_{h, i}\right)_{\Omega}+\frac{\partial a_{h}^{n}}{\partial \mathbf{U}_{h}}\left(\mathbf{U}_{h}^{n}, \boldsymbol{\psi}_{h, i}, \lambda_{h}^{n+1}\right) \\
& -\sum_{j=0, L}\left[\frac{\partial \Theta_{j}}{\partial \mathbf{U}_{h}}\left(\mathbf{U}_{h}^{n}\right)\left(\boldsymbol{\psi}_{h, i}, \mathbf{U}_{h, j}^{n+1}\right)-\frac{\partial \mathbf{T}_{j}}{\partial \mathbf{U}_{h}}\left(\mathbf{U}_{h}^{n}\right) \boldsymbol{\psi}_{h, i}\right] \cdot \mu_{z j, h}^{n+1} .
\end{aligned}
$$

The equations (40)-(41) and (42)-(43) consitute the adjoint problem associated with the direct problem (20). Some details concerning the derivatives that appeared in the equations (42)-(43), in particular a way to express analytically $\frac{\partial a_{h}^{n}}{\partial \mathbf{U}_{h}}$, will be given in a forecoming article.

Several remarks can be made.

Remark 3.2. First, one can note that equations at final time $t^{N_{t}+1}(40)-(41)$ are deduced from the general equations (42)-(43), with the assumption that $\lambda^{N_{t}+1} \equiv 0$ and $\mu_{z i, h}^{N_{t}+1} \equiv 0, i=0, L$.

Second, the adjoint problem is, as expected, linear in $\left(\lambda_{h}, \mu_{z 0, h}, \mu_{z L, h}\right)$ and backward in time. It requires the full knowledge of $\mathbf{U}_{h}$ at all instants and nodes. As our problem is 1-d, we can afford to store this information, and no special technique is required to solve the adjoint problem.

Third, the adjoint problem has the same form as the direct problem (20). It is essentially a 1-d linear hyperbolic problem, but as we used a Taylor-Galerkin discretization for the direct problem that contains (second order) parabolic terms, the formulation (42)-(43) contains also nontrivial second order terms. The generic function $G$ (or the cost function $J$ ) introduces a source term in the adjoint equation.

Fourth, the equations on the boundaries can be solved at each time step $n$, after the computation of the internal Lagrange multiplier $\lambda_{h}^{n}$. They involve the inversion of two $2 \times 2$ matrices that are the transpose of the ones in the direct systems (19).

\subsubsection{Discrete gradient equation}

At this stage, to compute the gradient, one has simply to apply the proposition 3.1. The equation (31) yields for a $\delta \mathbf{p} \in \mathbb{R}^{\mathcal{P}}$

$$
\begin{aligned}
<\nabla G, \delta \mathbf{p}>_{\mathbb{R}^{\mathcal{P}}}=\nabla_{\mathbf{p}} G\left(\mathbf{p}, M\left(\mathbf{U}_{h}\right)\right) \cdot \delta \mathbf{p}+\sum_{n=0}^{N_{t}-1}-\frac{\partial a_{h}^{n}}{\partial \mathbf{p}}\left(\mathbf{U}_{h}^{n}, \lambda_{h}^{n+1} ; \mathbf{p}\right) \cdot \delta \mathbf{p} \\
+\sum_{n=0}^{N_{t}-1} \sum_{i=0, L}\left[\frac{\partial \Theta_{i}}{\partial \mathbf{p}}\left(\mathbf{U}_{h}^{n}, \mathbf{p}\right) \mathbf{U}_{h, i}^{n+1}-\frac{\partial \mathbf{T}_{i}}{\partial \mathbf{p}}\left(\mathbf{U}_{h}^{n}, \mathbf{p}\right)\right] \mu_{z i, h}^{n+1} \cdot \delta \mathbf{p} .
\end{aligned}
$$

\section{NumERICAL RESULTS}

In this section, some first numerical results obtained with the parameter estimation method presented in the previous sections are shown. In this study, only synthetic data were used for the optimization and a single constant parameter was estimated: in this section, the parameter vector is defined as $\mathbf{p}=\beta \in \mathbb{R}$, where $\beta$ is the compliance given in the equation (6), such that $\boldsymbol{\beta}=\left(2 \rho A_{0} \beta, 1 / 2\right)$. In order to simplify the optimization for these preliminary tests, $\beta$ was supposed constant in space. 
After validating the computation of the discrete cost function gradient, the estimation method was tested using different data and different amounts of data, in order to evaluate the sensitivity of the method with respect to the information given.

\subsection{Computing the $3 d$ synthetic data}

The data used for the parameter estimation was computed from two different 3-d fluid structure interaction models.

The first simulation was made using a shell model for the structure $[2,12]$.

For the second simulation, the model used is implemented in the lifev code, [9]. A linear Venant Kirchoff model was used for the structure. The fluid was modeled by the incompressible Navier-Stokes equations for a Newtonian fluid. The interaction algorithm uses an exact Jacobian preconditioner, [7].
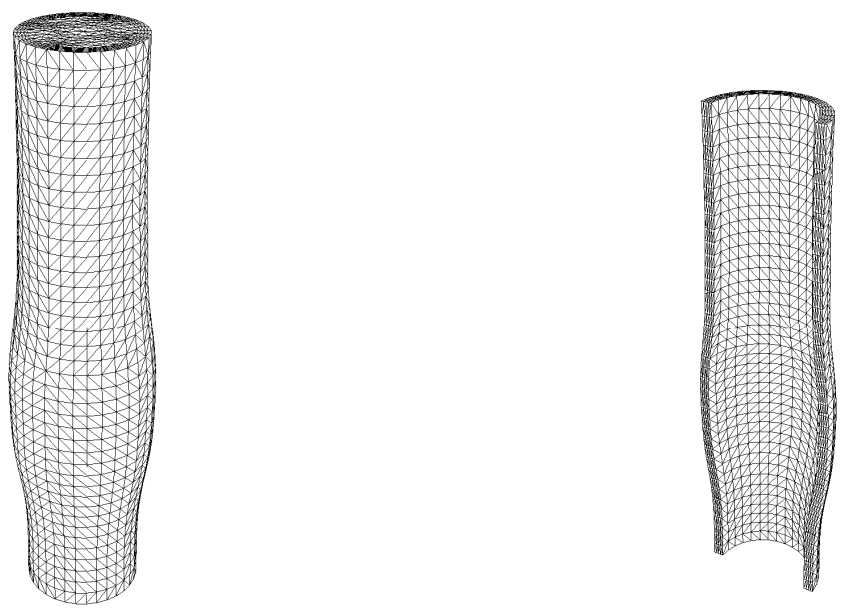

Figure 1. Left: fluid mesh. Right: structure mesh.

The domain used for both simulations is a cylindrical tube of length $L_{3 d}=5$ along the $z$ axis, with a circular basis of radius at rest $R_{0}=0.5$. A relatively coarse mesh was chosen for the simulation, as shown at Figure 1: the mesh is made of 30720 tetrahedra for the structural mesh and 68160 for the fluid mesh. The initial mesh is unstructured in the $O x y$ plane, but all nodes are contained in one of the 41 sections at altitude $z=i \times L_{3 d} / 40, i=0,1, \ldots, 40$.

The wall density $\rho_{w}$ was taken equal to 1.2 and the Poisson coefficient $\nu_{w}$ equal to 0.3 . Two different values of the Young modulus $E$ and the wall thickness at rest $h_{0, w}$ were used. For the first simulation, $E_{1}=3 . E 6$ and $h_{0, w}^{1}=0.05$, whereas for the second one $E_{2}=4 . E 6$ and $h_{0, w}^{2}=0.1$. The density of the fluid chosen was $\rho_{f}=1.0$ and its viscosity $\nu_{f}=0.03$.

The temporal discretization used was quite fine $(d t=1 E-4)$ in order to obtain a sufficient amount of data for the optimization. The number of timesteps computed was 99 for the first simulation and 79 for the second one. The simulations were stopped once the pressure wave arrived at the end of the tube, in order to avoid unphysiological reflexion.

The data obtained from each simulation was post-processed in order to have, at each time step and at each section of the tube, the values of the area $A d$, the flux $Q d$ and the pressure $P d$ of the blood flow (see the notations in section 3.1.4).

Figures 2 and 3 show two examples at the same instant of a 3-d model solution for two different Young modulus (the second is computed using a much coarser mesh, for visualizations purposes only). 

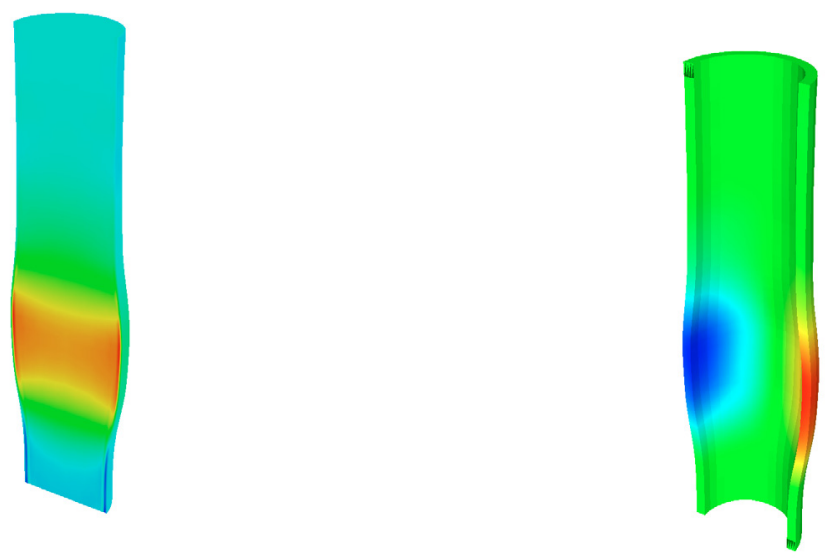

Figure 2. Solution of the 3 -d model at time $t=50 E-4$ for a Young modulus $E_{1}=4 E 6$. Left: fluid velocity along the $z$ axis. Right: displacement of the structure along $y$ axis.
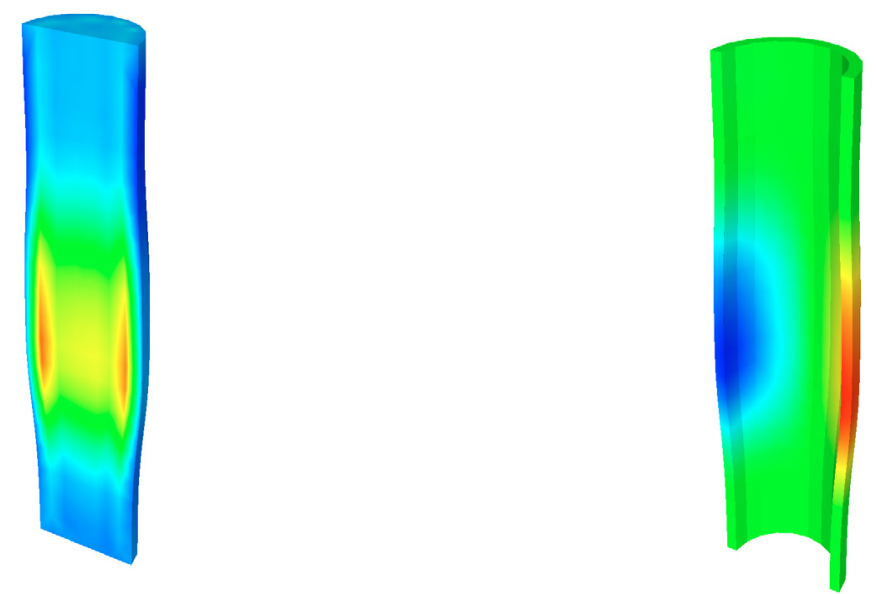

Figure 3. Solution of the 3 -d model at time $t=50 E-4$ for a Young modulus $E_{1}=6 E 6$. Left: fluid velocity along the $z$ axis. Right: displacement of the structure along $y$ axis.

One notes the effect when one increases the Young modulus: the pressure wave is extended in space and decreased in amplitude.

\subsection{Solving the 1-d-model}

In order to find the optimal value of a parameter, the algorithm does a series of simulations of the direct 1-d model.

The parameter estimated here was $\beta\left(=\frac{\beta_{0}}{2 \rho A_{0}}\right)$; all other parameters were kept constant: $\beta_{1}=1 / 2, \alpha=1$, $K_{r}=8 \pi \nu \approx 0.75, \rho=1, A_{0}=\pi R_{0}^{2} \approx 0.78$. 
The 1-d domain, denoted by $\Omega$, was chosen to be slightly shorter than the cylindrical tube used for the 3-d simulations: $\Omega=[0.5,4.5]$, and its length was therefore $L=4$. This was done in order to avoid the problems arising from the difference in the type of the boundary conditions between the 3-d and the 1-d models.

The 1-d computations were made with a spatial discretization of $N=128$ elements of constant length $L / N=4 / 128$, and a time step $d t=1 . e-5$, that is ten times smaller than the 3 -d time step. These values ensure the Courant condition to be always respected.

\subsection{Measurement operator and cost function}

For each test case, a series of estimations of $\beta$ was done using different amounts of data. This means that the measurement operator used for the optimization was changed, and so was, consequently, the expression of the cost function $J$.

As explained in section 3.1.2, the measurement operator is a weighted sampling of the direct solution expressed by (26). The weights used here were $w^{A} \equiv 1$ and $w^{Q} \equiv 0.1$ for all measures. They were chosen to compensate the orders of magnitude of the area $(\mathcal{O}(1))$ and the flux $(\mathcal{O}(10))$ in the vessel. Three different space samplings were chosen: the first one uses 33 measures taken at $\zeta_{j}=0.5+j \times(4 / 32)$, for $j=0, \ldots, 32$. The second one uses 3 measures, at $\zeta_{1}=0.5, \zeta_{2}=2.5$ and $\zeta_{3}=4.5$, and the last one only two, at $\zeta_{1}=0.5$ and $\zeta_{2}=4.5$. The time sampling consists in using the data at either all the instants available or only at every ten of them. For the first simulation, that means the measures are taken at $\theta_{\nu}=\nu \times d t$ for $\nu=0, \ldots, 99$, or at $\theta_{\nu}=10 \nu \times d t$ for $\nu=0, \ldots, 9$. For the second simulation, they are taken at $\theta_{\nu}=\nu \times d t$ for $\nu=0, \ldots, 79$ or at $\theta_{\nu}=10 \nu \times d t$ for $\nu=0, \ldots, 7$.

Let us note that the cost function minimized during each estimation is normalized. Its expression is the following (cf. (29)):

$$
J(\beta)=\frac{\sum_{\nu=0}^{\mathcal{V}_{t}} \sum_{j=0}^{\mathcal{V}_{z}}\left\{\left(A d_{j}^{\nu}-A_{\phi_{z}(j)}^{\phi_{t}(\nu)}\right)^{2}+(0.1)^{2}\left(Q d_{j}^{\nu}-Q_{\phi_{z}(j)}^{\phi_{t}(\nu)}\right)^{2}\right\}}{\sum_{\nu=0}^{\mathcal{V}_{t}} \sum_{j=0}^{\mathcal{V}_{z}}\left\{\left(A d_{j}^{\nu}\right)^{2}+(0.1)^{2}\left(Q d_{j}^{\nu}\right)^{2}\right\}} .
$$

\subsection{Validating the computation of the cost function gradient}

In order to validate the computation of the cost function gradient using the method presented here, its value was compared with the value obtained with the finite difference method. This method consists in computing an approximation of the derivative of the cost function $J(\beta)$ with respect to $\beta$, that is:

$$
\frac{\partial J}{\partial \beta}(\beta)=\lim _{h \rightarrow 0} \frac{J(\beta+h)-J(\beta)}{h}
$$

The cost function gradient was computed in some test cases with both methods and the results, made in double precision, showed 6 to 7 identical numbers.

\subsection{Expected results of the estimation}

Let us recall the role of the parameter $\beta$. As exposed in section 2, to derive the 1-d blood model from the 3 -d one, a wall displacement law (cf. (4)) is used, that introduces two positive real parameters $\beta_{0}$ and $\beta_{1}$. In these tests, the power coefficient $\beta_{1}$ is taken equal to $1 / 2$ and the law is reformulated into the expression (6), that only involves one positive parameter $\beta$.

A linear elastic law provides the following expression for $\beta$ :

$$
\beta_{3 d}=\frac{\sqrt{\pi} h_{0, w} E}{2 \rho A_{0}\left(1-\nu_{w}^{2}\right)},
$$


where $E, h_{0, w}$ and $\nu_{w}$ are the wall Young modulus, the wall thickness at rest and the Poisson coefficient used to compute the 3-d data.

The optimal value of $\beta$, minimizing the difference between the results of the 1-d and 3-d models, is therefore expected to be $\beta_{3 d}$. It will be shown that this is not the case: the results obtained computing the 1-d model with the estimated optimal value, $\beta_{\text {estim }}$, fit better to the $3 \mathrm{~d}$ data than the results obtained with $\beta_{3 d}$. The accuracy of the estimation algorithm is therefore not to be evaluated from the accuracy on $\beta$, but from the accuracy on the error between the 1-d results and the data.

\subsection{Test case 1: shell structure, $E=3 . E 6$ and $h_{0, w}=0.05$}

The first test was made using the 3-d data obtained with a shell / Navier-Stokes coupling. The structure has a Young modulus equal to $3 . E 6$ and a wall thickness equal to 0.05 . The number of iterations needed for the optimization varied between 6 and 10 for all the different estimations.

\subsubsection{Results of the optimization}

The results obtained are shown in tables 1 and 2: for each estimation, the optimal value $\beta_{\text {estim }}$ is given, as well as the corresponding value of the minimized cost function $J$ and the value $1-\sqrt{J}$, that gives the order of explained data.

In order to better compare the precision of the different estimations, we also give the value of a general cost function, called "fine cost function", and denoted by $J_{\text {fine }}$. This function $J_{\text {fine }}$ computes the least squares error between the 3-d data measured at each of the 99 timesteps and the 33 sections, and the 1-d data obtained with the estimated value $\beta_{\text {estim }}$.

\begin{tabular}{||c|c|c|c||}
\hline $\mathrm{Nb} z_{\text {meas }}$ & 33 & 3 & 2 \\
\hline$\beta_{\text {estim }}$ & $1.3 E 5$ & $1.32 E 5$ & $1.41 E 5$ \\
\hline$J$ & 0.048 & 0.027 & $1.6 E-5$ \\
\hline $1-\sqrt{J}$ & $78.1 \%$ & $83.4 \%$ & $99.6 \%$ \\
\hline$J_{\text {fine }}$ & 0.048 & 0.048 & 0.052 \\
\hline $1-\sqrt{J_{\text {fine }}}$ & $78.1 \%$ & $78 \%$ & $77.2 \%$ \\
\hline
\end{tabular}

TABLE 1. Results using 99 time measurements and 33, 3 or 2 spatial measurements for a 3 -d data such that: $E=3 E 6$ and $h_{0, w}=0.05$.

\begin{tabular}{||c|c|c|c||}
\hline $\mathrm{Nb} z_{\text {meas }}$ & 33 & 3 & 2 \\
\hline$\beta_{\text {estim }}$ & $1.32 E 5$ & $1.33 E 5$ & $1.41 E 5$ \\
\hline$J$ & 0.038 & 0.02 & $3 E-6$ \\
\hline $1-\sqrt{J}$ & $80.6 \%$ & $85.9 \%$ & $99.8 \%$ \\
\hline$J_{\text {fine }}$ & 0.048 & 0.048 & 0.052 \\
\hline $1-\sqrt{J_{\text {fine }}}$ & $78.0 \%$ & $78.0 \%$ & $77.2 \%$ \\
\hline
\end{tabular}

TABLE 2. Results using 9 time measurements and 33, 3 or 2 spatial measurements for a 3 -d data such that: $E=3 E 6$ and $h_{0, w}=0.05$. 
We can see that, logically, the most accurate estimation is obtained in the case where the biggest amount of 3 -d data is used for the optimization. But the results do not degenerate too much when the number of time or space measurements is reduced. In fact, the value of $\beta$ estimated from only 9 time and 2 space measurements, provides results that explain $77,2 \%$ of the data.

The results are actually quite stable with respect to the amount of data used. Particularly, when the number of time measures varies from 99 to 9 , the estimated value of $\beta$ remains almost constant. On the other hand, we notice that the value of $\beta_{\text {estim }}$ is more sensitive to the variation of the number of space measures. But this sensitivity remains relatively low, the maximal variation of $\beta_{\text {estim }}$ being of $10 \%$, when the amount of space measurements changes from 33 to 2 .

\subsubsection{Results of the 1-d model using the estimated value $\beta_{\text {estim }}$}

For this test case, the expected value of $\beta$, according to relation (46), is $\beta_{3 d}=1.87 E 5$. In order to compare this value with the results of the different estimations, the 1-d model has also been computed using $\beta_{3 d}$. The percentage of explained data obtained with this simulation, measured with the function $1-\sqrt{J_{\text {fine }}}$, is of $64 \%$, which is far bellow the one obtained with the different estimations made (see tables 1 and 2 ).

We show next the results of the 1 -d model computed using the estimated value $\beta_{\text {estim }}=1.30 E 5$, obtained from 99 time and 33 space measurements of the 3 -d data. The results are compared with the 3 -d data and with the 1-d results computed using $\beta_{3 d}$. Note that there is a difference of about $30 \%$ between the values $\beta_{\text {estim }}$ and $\beta_{3 d}$. Figures 4 and 5 represent the area of the vessel and the flux of the blood flow in the whole domain, after 50 and 90 time steps. Figure 6 shows the time evolution of the area and the flux at the middle of the tube, that is at $z=L / 2$.
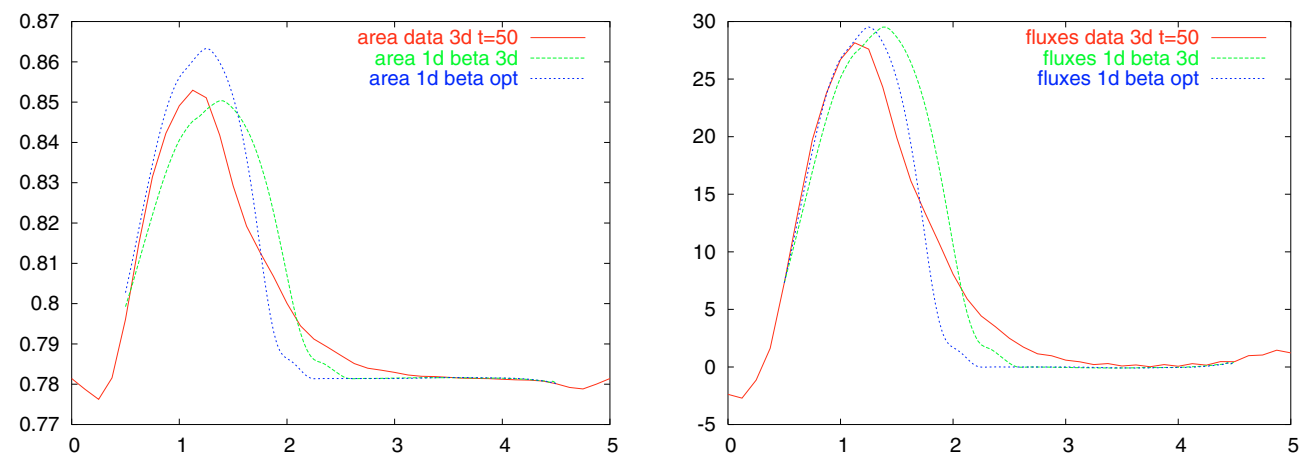

Figure 4. Area and flux obtained from the 1-d model using $\beta_{\text {estim }}$ (blue dotted line) and $\beta_{3 d}$ (green dashed line), compared to the 3-d data (red line), after 50 timesteps.

As an additional information, the pressure of the blood flow was computed from the 1-d results according to the wall displacement law (6). It is displayed after 50 and 90 time steps in Figure 7.

On these figures, we observe that the results of the 1-d model manage to capture quite well the phase of the waves of the 3-d data, but not its shape and amplitude. Particularly, the waves of the 3-d data are significantly larger. This can be explained by one of the assumptions made for the derivation of the 1-d model, that considers the vessel as a sequence of independent rings. On the contrary, the 3 -d model used to compute the data takes into account the propagation in the structure. On the other hand, the difference in the amplitude can be due to the more important diffusivity of the 3 -d models.

In addition, we can see that the results obtained with the estimated optimal value $\beta_{\text {estim }}$ are closer to the 3 -d data than those computed using the expected value $\beta_{3 d}$. This becomes particularly true after 90 timesteps and at the middle of the tube, where the waves obtained with $\beta_{3 d}$ are clearly in advance with respect to the data. On the contrary, the phase error of the waves obtained with $\beta_{\text {estim }}$ remains relatively small. 

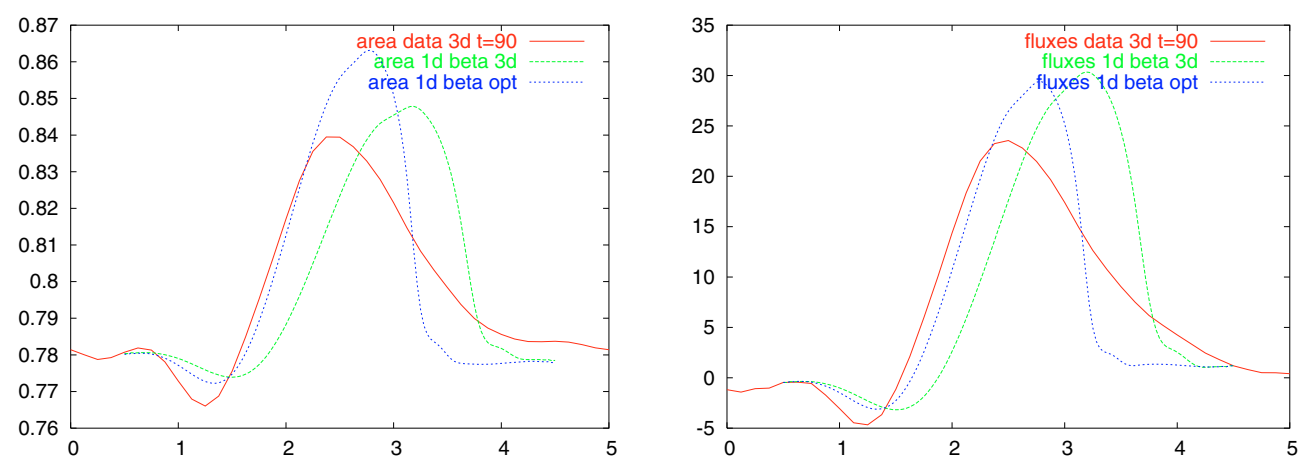

Figure 5. Area and flux obtained from the 1-d model using $\beta_{\text {estim }}$ (blue dotted line) and $\beta_{3 d}$ (green dashed line), compared to the 3-d data (red line), after 90 timesteps.
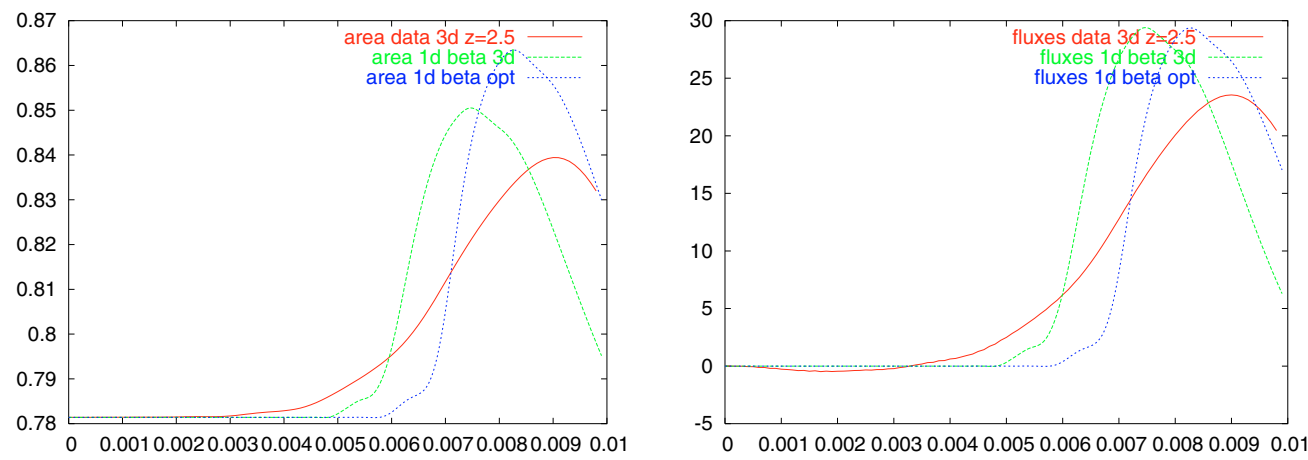

Figure 6. Area and flux obtained from the 1-d model using $\beta_{\text {estim }}$ (blue dotted line) and $\beta_{3 d}$ (green dashed line), compared to the 3 -d data (red line), at $z=L / 2$.
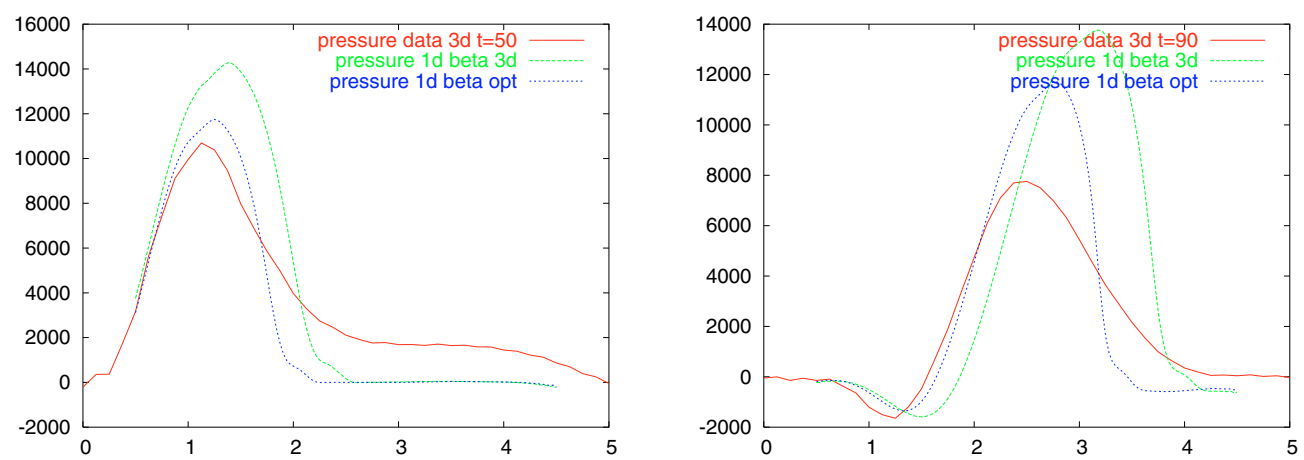

Figure 7. Pressure obtained from the 1 -d model using $\beta_{\text {estim }}$ (blue dotted line) and $\beta_{3 d}$ (green dashed line), compared to the 3-d data (red line). Left: after 50 timesteps. Right: after 90 timesteps.

This result has to be emphasized, because it proves the existence of a more optimal value of $\beta$ than the one given by (46), a relation resulting from physical considerations. 


\subsubsection{Sensitivity of the estimation with respect to the space measurements}

Next, the optimal values $\beta_{\text {estim }}$ obtained from different amounts of space measurements are compared, the number of time measurements being constant and equal to 99 . The results of the 1-d model computed with these values are shown in figures (8) and (9). The comparison between the values estimated from 9 time measures is not made because, as deduced from tables 1 and 2 , there is almost no difference.
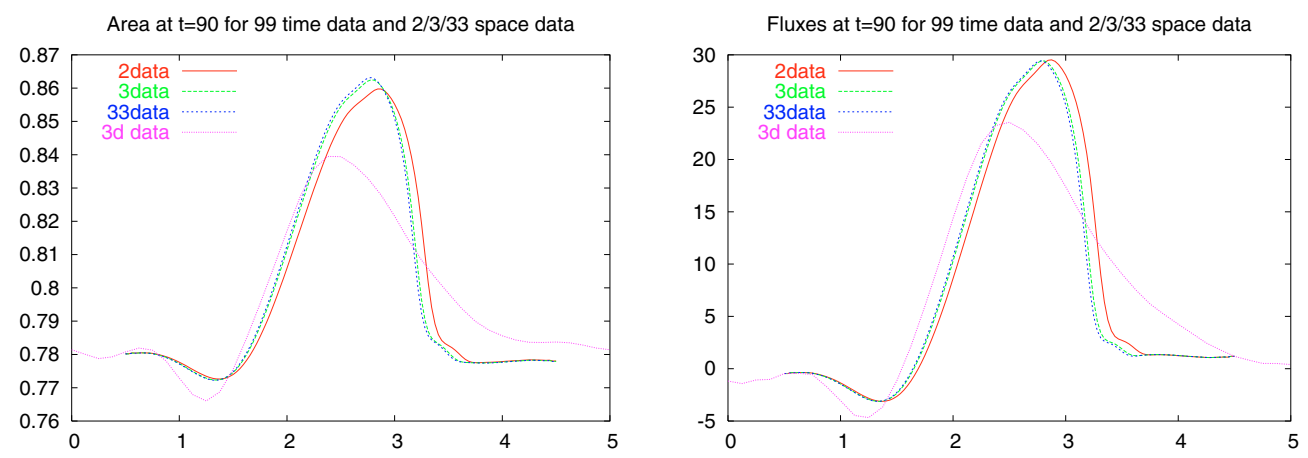

Figure 8. Results of the 1-d model using the value $\beta_{\text {estim }}$ obtained from 99 time measurements and 33 (blue dotted line), 3 (green dashed line) or 2 (red line) space measurements, compared to the 3 -d data (mauve small-dotted line), after 90 timesteps.
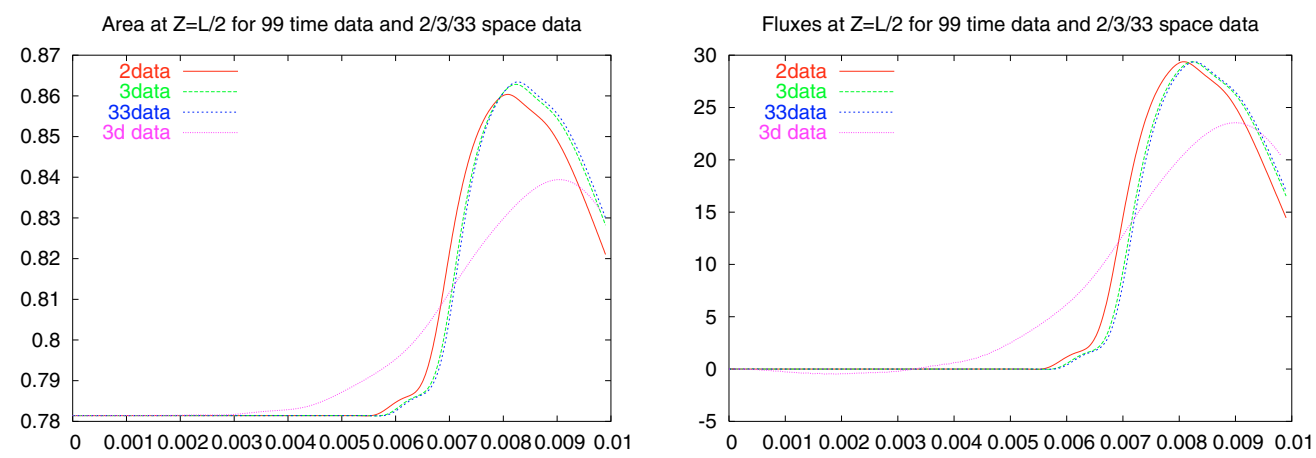

Figure 9. Results of the 1-d model using the value $\beta_{\text {estim }}$ obtained from 99 time measurements and 33 (blue dotted line), 3 (green dashed line) or 2 (red line) space measurements, compared to the 3 -d data (mauve small-dotted line), at $z=L / 2$.

We can see that the optimal values of $\beta$ estimated from 33 and 3 space measures provide almost the same 1-d results. But when changing to 2 space measurements, the phase error of the 1-d results grows significantly, so that the advance of the area and flux waves with respect to the data increases largely. This means that with 2 space measurements, the estimation is not yet stable.

\subsubsection{Sensitivity of the estimation with respect to the time measurements}

The comparison is now made between the optimal values of $\beta$ obtained from 99 and 9 time measurements, the number of space measurements being constant and equal to 2 , which is the minimum. The results of the 1-d model computed with these values are shown in figures (10) and (11). 

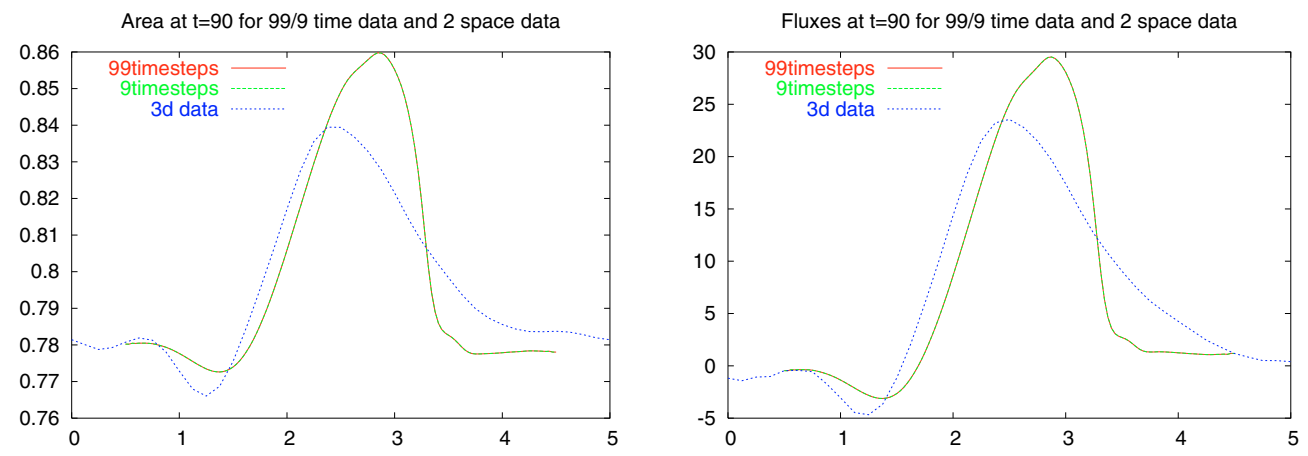

Figure 10. Results of the 1-d model using the value $\beta_{\text {estim }}$ obtained from 2 space measurements and 99 (blue dotted line) or 9 (red line) time measurements, compared to the 3-d data (mauve small-dotted line), after 90 timesteps. The two curves with 9 and 99 are almost identical.
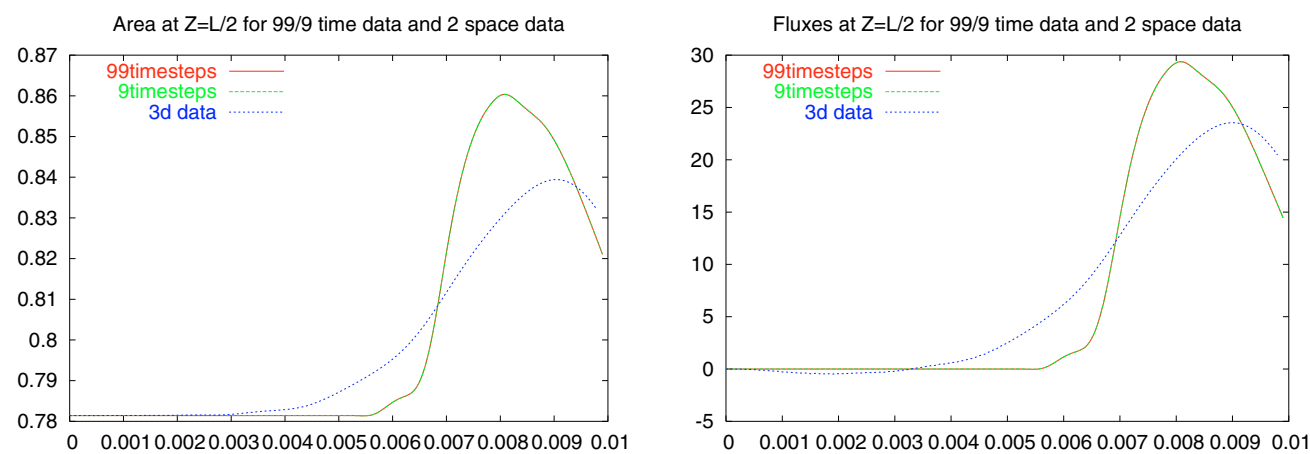

Figure 11. Results of the 1-d model using the value $\beta_{\text {estim }}$ obtained from 2 space measurements and 99 (blue dotted line) or 9 (red line) time measurements, compared to the 3-d data (mauve small-dotted line), at $z=L / 2$. The two curves with 9 and 99 are almost identical.

We observe that, in both cases, the curves are perfectly superposed. The quantity of explained data is thus already stable at 9 time measures.

\subsection{Test case 2: 3-d structure, $E=4 . E 6$ and $h_{0, w}=0.1$}

The second test was made using the 3 -d data obtained with a 3-d structure model with a Young modulus of $4 . E 6$ and a wall thickness of 0.1 . Here again, the number of iterations necessary for the optimization was bounded by 6 and 10 .

\subsubsection{Results of the optimization}

The results obtained are shown in tables 3 and 4 . The fine cost function computes here the least squares error between the 3 -d data measured at each of the 79 timesteps and the 33 sections, and the 1-d data obtained with the estimated value $\beta_{\text {estim }}$.

As expected, the most accurate estimation is obtained with the biggest amount of 3 - $\mathrm{d}$ data, that is 79 time and 33 space measurements. The percentage of explained data, measured with the function $1-\sqrt{J_{\text {fine }}}$, is in this case of $88.8 \%$. But in the case where only 7 time and 2 space measurements are used, this percentage is 


\begin{tabular}{||c|c|c|c||}
\hline Nb $z_{\text {meas }}$ & 33 & 3 & 2 \\
\hline$\beta_{\text {estim }}$ & $3.13 E 5$ & $3.20 E 5$ & $3.67 E 5$ \\
\hline$J$ & 0.013 & 0.0049 & $1.4 E-5$ \\
\hline $1-\sqrt{J}$ & $88.8 \%$ & $93 \%$ & $99.6 \%$ \\
\hline$J_{\text {fine }}$ & $i d e m$ & 0.013 & 0.025 \\
\hline $1-\sqrt{J_{\text {fine }}}$ & $i d e m$ & $88.6 \%$ & $84.1 \%$ \\
\hline
\end{tabular}

TABLE 3. Results using 79 time measurements and 33, 3 or 2 spatial measurements for a $3-\mathrm{d}$ data such that: $E=4 E 6$ and $h_{0, w}=0.1$.

\begin{tabular}{||c|c|c|c||}
\hline Nb $z_{\text {meas }}$ & 33 & 3 & 2 \\
\hline$\beta_{0, \text { estim }}$ & $5.64 E 5$ & $5.74 E 5$ & $5.98 E 5$ \\
\hline$\beta_{\text {estim }}$ & $3.19 E 5$ & $3.24 E 5$ & $3.38 E 5$ \\
\hline$J$ & 0.0087 & 0.0017 & $1.1 E-5$ \\
\hline $1-\sqrt{J}$ & $90.7 \%$ & $95.9 \%$ & $99.7 \%$ \\
\hline$J_{\text {fine }}$ & 0.013 & 0.013 & 0.016 \\
\hline $1-\sqrt{J_{\text {fine }}}$ & $88.7 \%$ & $88.4 \%$ & $87.4 \%$ \\
\hline
\end{tabular}

TABLE 4. Results using 7 time measurements and 33, 3 or 2 spatial measurements for a $3-\mathrm{d}$ data such that: $E=4 E 6$ and $h_{0, w}=0.1$.

of $87.4 \%$, which is not much lower. Here again, the accuracy of the estimation does not decrease significantly with the amount of data.

But the results of the estimation are less stable than in the first case. Particularly, the variation of the estimated value when the time sampling changes is quite important. Indeed, when only 2 space measurements are considered, the relative variation of $\beta_{\text {estim }}$ reaches $17 \%$ when the amount of time measurements is decreased from 79 to 7 .

\subsubsection{Results of the 1-d model using the estimated value $\beta_{\text {estim }}$}

For this test case, the expected value of $\beta$, according to the relation (46), is $\beta_{3 d}=4.96 E 5$. This value provides 1-d results that explain $71 \%$ of the data, which is again far bellow the percentage obtained with the different estimations made (see tables 3 and 4 ). Note that the maximal difference between the estimated value $\beta_{\text {estim }}$ and $\beta_{3 d}$ is of about $38 \%$.

Next, we show the results of the 1 -d model, computed using the estimation $\beta_{\text {estim }}=3.13 E 5$, which provides the highest value of $1-\sqrt{J}$. They are compared with the 3 -d data and with the 1 -d results computed using $\beta_{3 d}$. Figures 12 and 13 represent the area of the vessel and the flux the blood flow in the whole domain, after 50 and 70 time steps. Figure 14 shows the time evolution of the area and the flux at the middle of the tube, that is at $z=L / 2$.

Firstly, we observe that in this case the results of the 1-d model fit much better to the 3-d data than in the test case one. In fact, not only the phase of the waves is captured, but their shape and amplitude is also well approached. Let us note that the 3 -d model used here to compute the data is linear for the structure. This is probably the reason why, in this case, the linear 1 -d model is closer to the 3 -d one. 

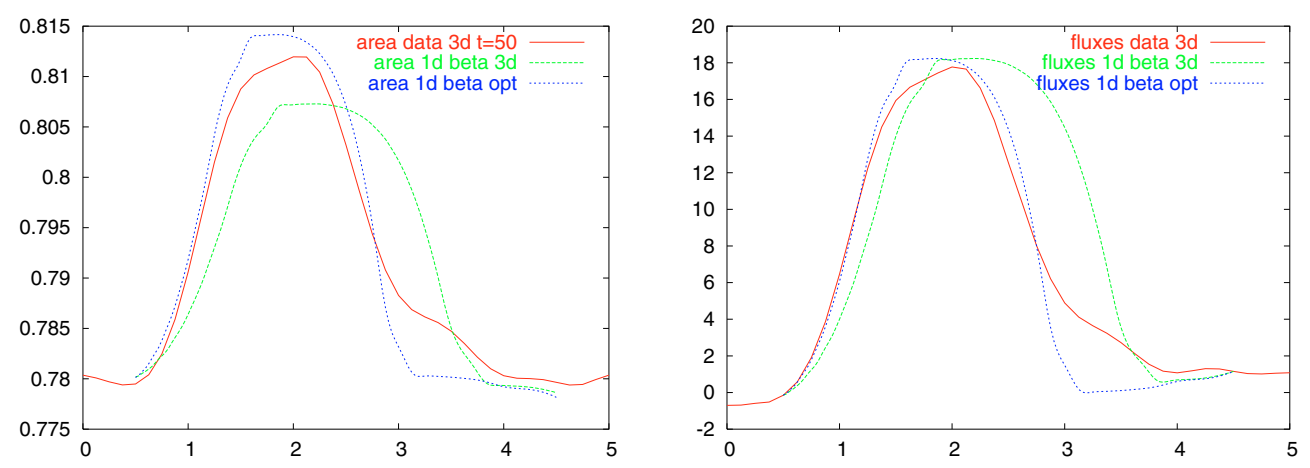

Figure 12. Results of the 1 -d model using $\beta_{\text {estim }}$ (blue dotted line) and $\beta_{3 d}$ (green dashed line), compared to the 3 -d data (red line), after 50 timesteps.
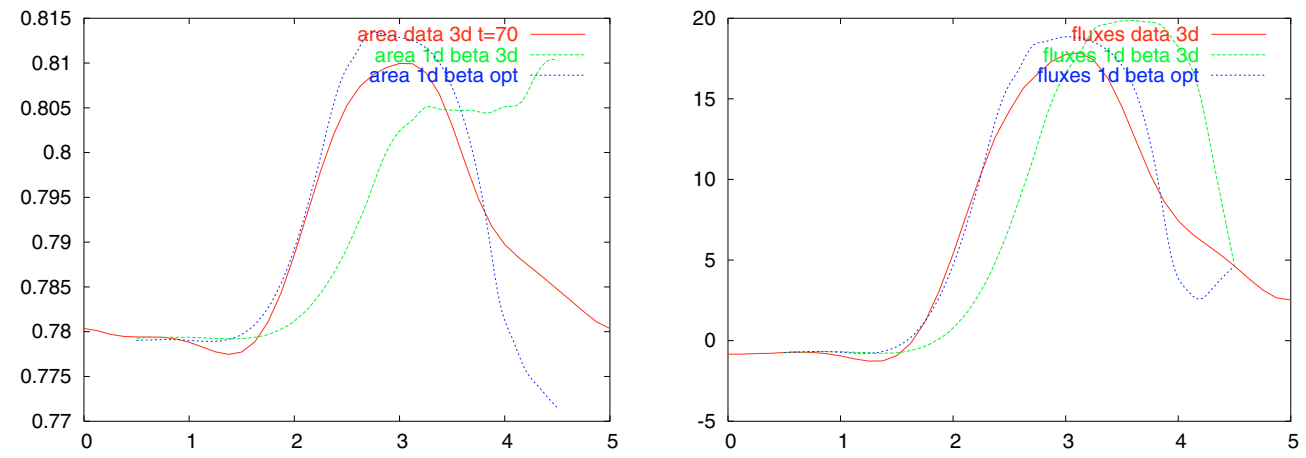

Figure 13. Results of the 1 -d model using $\beta_{\text {estim }}$ (blue dotted line) and $\beta_{3 d}$ (green dashed line), compared to the 3 -d data (red line), after 70 timesteps.
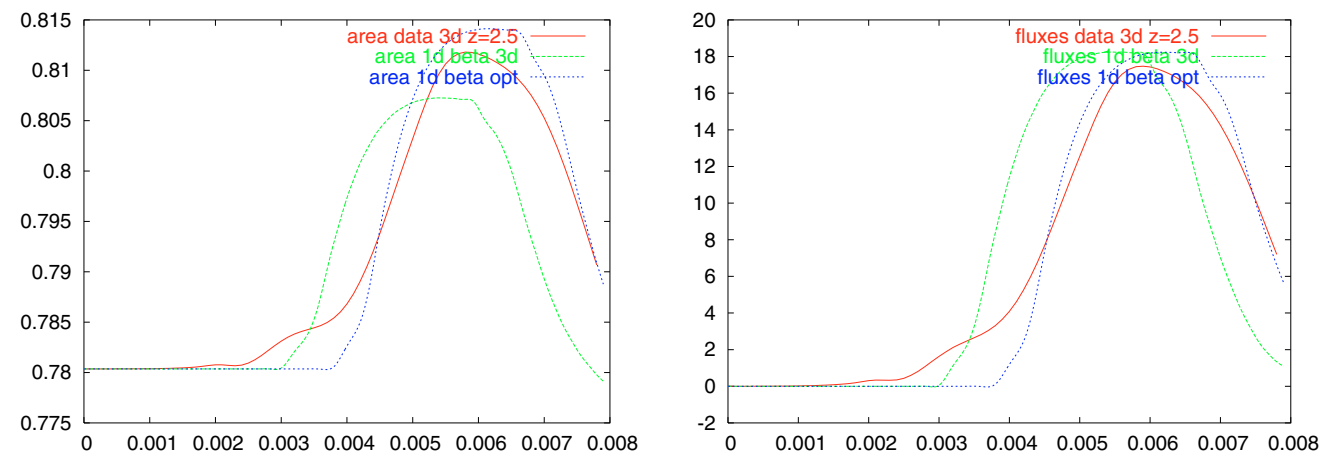

Figure 14. Results of the 1-d model using $\beta_{\text {estim }}$ (blue dotted line) and $\beta_{3 d}$ (green dashed line), compared to the 3 -d data (red line), at $z=L / 2$.

We can also see that the results obtained with the value $\beta_{\text {estim }}$ are much better than those obtained with $\beta_{3 d}$. Indeed, the waves obtained with this last value are much too advanced with respect to the data, whilst the phase error of the waves obtained with $\beta_{\text {estim }}$ remains relatively small, even after 70 timesteps. 


\subsubsection{Sensitivity of the estimation with respect to the space measurements}

The optimal values $\beta_{\text {estim }}$ obtained from different amounts of space measurements are now compared, the number of time measurements being fixed at 79. The results of the 1-d model computed with these values are shown in figures (15) and (16).
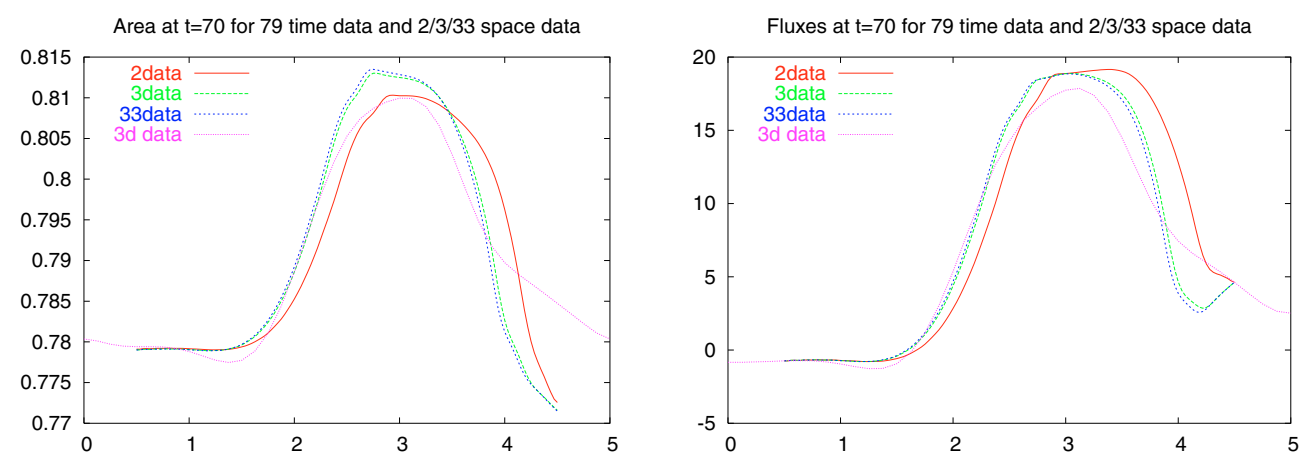

Figure 15. Results of the 1-d model using the value $\beta_{\text {estim }}$ obtained from 79 time measurements and 33 (blue dotted line), 3 (green dashed line) or 2 (red line) space measurements, compared to the 3 -d data (mauve small-dotted line), after 70 timesteps.
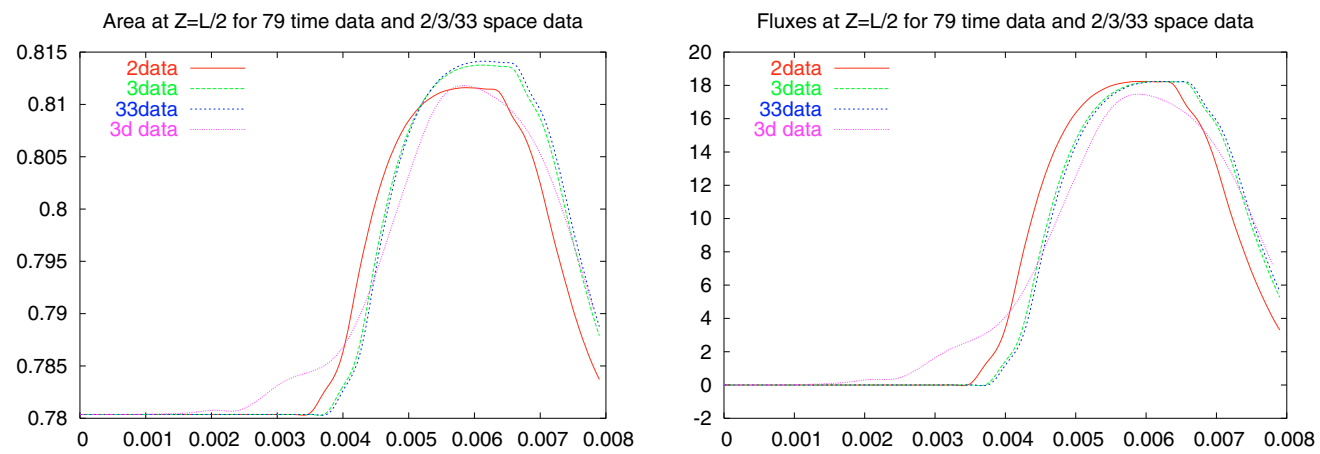

Figure 16. Results of the 1 -d model using the value $\beta_{\text {estim }}$ obtained from 79 time measurements and 33 (blue dotted line), 3 (green dashed line) or 2 (red line) space measurements, compared to the 3 -d data (mauve small-dotted line), at $z=L / 2$.

These figures show that, here again, a significant variation in the 1-d results only occures when dicreasing the amount of space measurements from 3 to 2 . In that case, the phase of the waves is lost, so that the area and flux appear to be much more in advance with respect to the data. Thus, we can say that the estimation starts to be stable from 3 space measurements.

\subsubsection{Sensitivity of the estimation with respect to the time measurements}

Here, the comparison is made between the estimated values of $\beta$ obtained using 79 and 7 time measurements. The amount of space measurements is first fixed at 33 and later at 2 . The results of the first comparison are shown in figures 17 and 18. For the second one, the results are shown in figures 19 and 20.

From the observation of these figures, we can say that, when 33 space measurements are used, the estimation is stable with respect to the amount of time measurements. On the contrary, when the data is only considered 

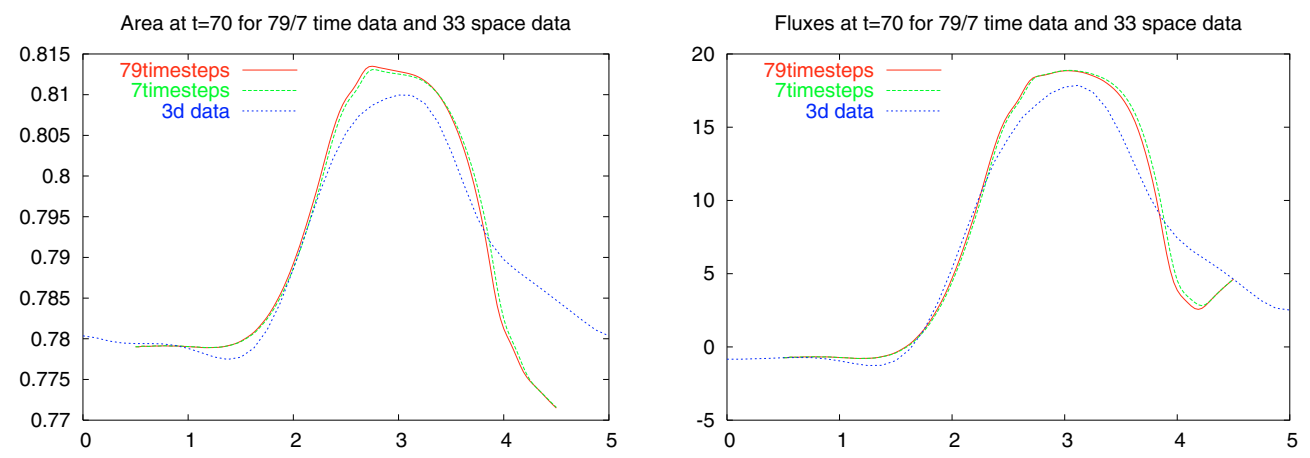

Figure 17. Results of the 1 -d model using the value $\beta_{\text {estim }}$ obtained from 33 space measurements and 79 (blue dotted line) or 7 (red line) time measurements, compared to the 3-d data (mauve small-dotted line), after 70 timesteps.
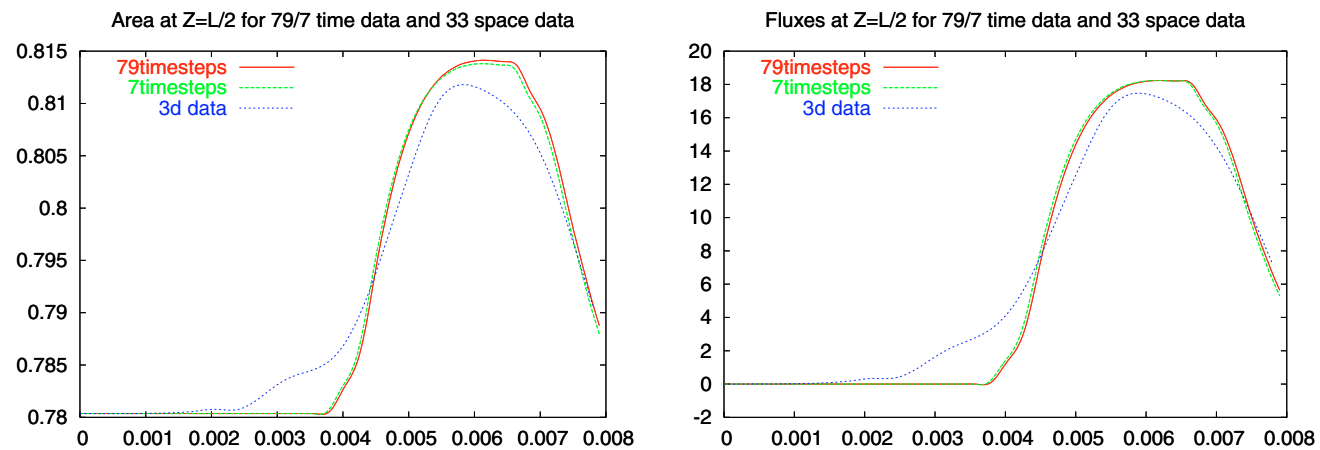

FigurE 18. Results of the 1 -d model using the value $\beta_{\text {estim }}$ obtained from 33 space measurements and 79 (blue dotted line) or 7 (red line) time measurements, compared to the 3-d data (mauve small-dotted line), at $z=L / 2$.

at 2 points in space, the estimation is unstable and varies significantly with the amount of time measurements provided.

These numerical tests show that it is possible to identify the parameter $\beta$ in a stable way from 3 measures in space and 7 measures in time (in the first test case already from 2 measures in space and 9 in time). Moreover, the estimations obtained are more optimal than the expected value $\beta_{3 d}$, as they provide 1 - $d$ results that explain a bigger percentage of data.

\section{Conclusions}

In this work, the parameter identification of a 1-d blood flow model using the adjoint approach was studied. Starting from the discretization of the model with a second order Taylor-Galerkin scheme, the discrete adjoint problem was derived. We obtained a linear 1-d hyperbolic system, with non-standard discretization and boundary conditions. The resolution of the adjoint problem allows to compute the gradient of a cost function, which evaluates the least squares error between the measured data and the 1-d results. The analytical discrete gradient is necessary to minimize the cost function in order to find the optimal values of the parameters. Its computation was therefore implemented and validated using the finite difference method. Some preliminary 

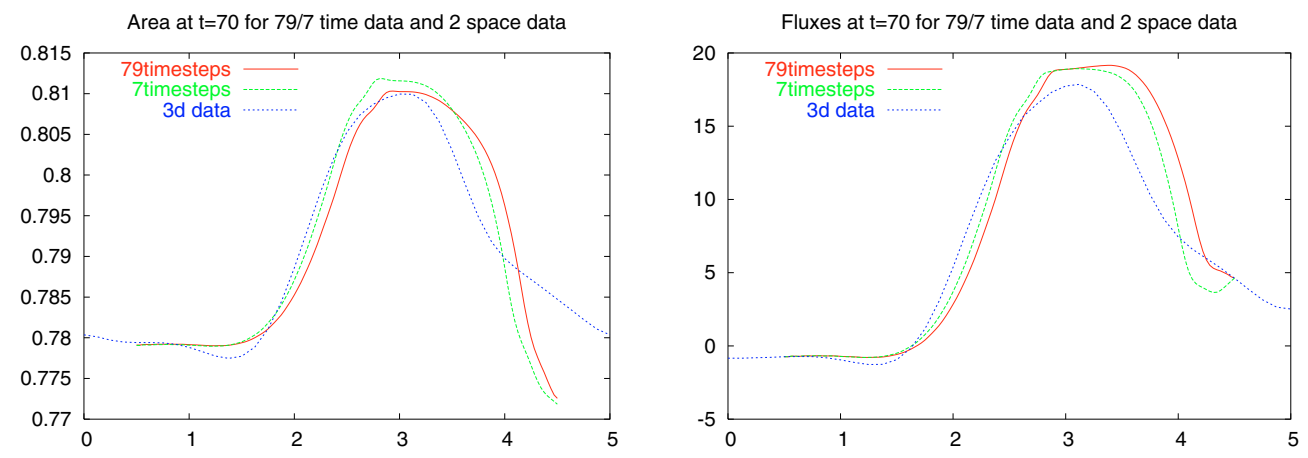

Figure 19. Results of the 1-d model using the value $\beta_{\text {estim }}$ obtained from 2 space measurements and 79 (blue dotted line) or 7 (red line) time measurements, compared to the 3-d data (mauve small-dotted line), after 70 timesteps.
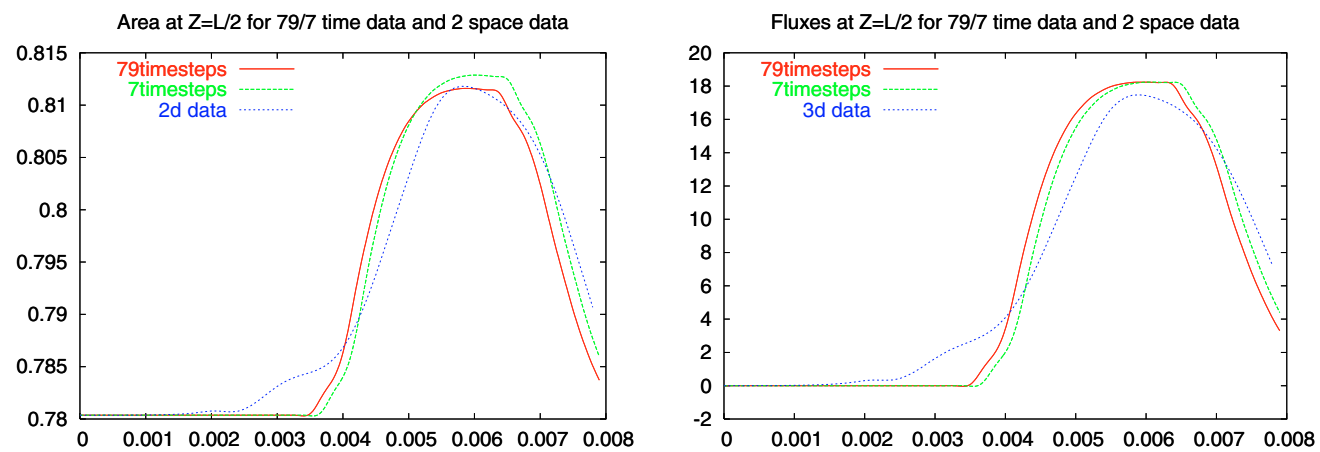

Figure 20. Results of the 1-d model using the value $\beta_{\text {estim }}$ obtained from 2 space measurements and 79 (blue dotted line) or 7 (red line) time measurements, compared to the 3-d data (mauve small-dotted line), at $z=L / 2$.

numerical tests were performed. A single parameter was estimated - the arterial compliance $\beta$ - which is the most important parameter from a biomedical point of view. In order to simplify the estimation, $\beta$ was assumed to be constant in space. The optimization was made using data provided by two different 3-d models, and for two different values of the Young modulus and the wall thickness at rest. In all cases, the estimated values of $\beta$ obtained were very different from the expected value $\beta_{3 d^{1}}$, the difference going from 30 to $38 \%$. However, the 1-d results computed with the estimated values appeared to be significantly closer to the data than those obtained with $\beta_{3 d}$. Actually, the percentage of explained data obtained with these estimations was of about $75 \%$ to $89 \%$, depending on the test case and the amount of data used for the optimization (this is not surprising as in the first case the data were computed using a shell model with a non-linear constitutive law). Thus, it is possible to retrieve the pressure of the blood flow in an artery in a non-intrusive way.

The interest of this work first lies in the application of a parameter estimation method to a 1-d blood flow model, using a rigorous mathematical approach. Second, the method used for the optimization is efficient. Indeed, once the adjoint state has been derived from the discrete 1-d model, the estimation can quite simply be extended to more parameters and especially to parameters depending on space.

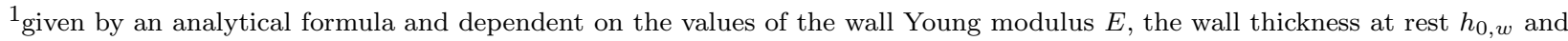
the Poisson coefficient $\nu_{w}$.
} 
This work is a first step in the parameter identification for the 1-d blood flow model. The tests were for the moment limited to the estimation of a single parameter, assumed to be constant in space and time. The optimization should now be extended to other parameters, possibly depending on space. After a sensitivity analysis, it would be interesting to estimate the area at rest $A_{0}$ at each discretization point of the 1-d domain, because this could allow to localize a possible tapering in the vessel. Another relevant application of the method would be the parameter estimation involved in a bifurcation.

\section{REFERENCES}

[1] D. Ambrosi and L. Quartapelle. A Taylor-Galerkin method for simulating nonlinear dispersive water waves. J. Comput. Phys., 146(2):546-569, 1998.

[2] D. Chapelle and K. J. Bathe. The finite element analysis of shell - Fundamentals. Springer-Verlag, 2004.

[3] G. Chavent. Non linear Least Squares for Inverse problems. 2005. in preparation.

[4] Guy Chavent. Identification of distributed parameter systems : about the output least square method, its implementation and identifiability. In R. Isermann, editor, Identification and system parameter identification (Proc. Fifth IFAC Symposium on Identification, Darmstadt), pages 85-97. Pergamon Press, Amsterdam, 1979.

[5] Guy Chavent. On the theory of practice of nonlinear least-squares. Adv. in Water Res., 14(2):55-63, 1991. Parameter identification in ground water flow, transport, and related processes, Part I.

[6] F. Clément, N. Khvoenkova, A. Cartalade, and Montarnal Ph. Analyse de sensibilit et estimation de paramtres de transport pour une quation de diffusion, approche par tat adjoint. Report 5132, Inria, Rocquencourt, France, 2004.

[7] Miguel Ángel Fernández and Marwan Moubachir. A newton method using exact jacobians for solving fluid-structure coupling. Computers and Structures, 83(2-3):127-142, 2005.

[8] L. Formaggia, J. F. Gerbeau, F. Nobile, and A. Quarteroni. On the coupling of 3D and 1D Navier-Stokes equations for flow problems in compliant vessels. Comput. Methods Appl. Mech. Engrg., 191(6-7):561-582, 2001.

[9] L. Formaggia, J.F. Gerbeau, and Prud'homme C. Lifev developer manual. Technical report, 2004. Software and documentation available electronically, (http://www.lifev.org/documentation/lifev-dev.pdf).

[10] Luca Formaggia, Daniele Lamponi, and Alfio Quarteroni. One-dimensional models for blood flow in arteries. J. Engrg. Math., 47(3-4):251-276, 2003. Mathematical modelling of the cardiovascular system.

[11] Luca Formaggia and Alessandro Veneziani. Reduced and multiscale models for the human cardiovascular system. Report 21, Mox, Politecnico di Milano, 2003.

[12] Jean-Frédéric Gerbeau and Marina Vidrascu. A quasi-Newton algorithm based on a reduced model for fluid-structure interaction problems in blood flows. M2AN Math. Model. Numer. Anal., 37(4):631-647, 2003.

[13] Mihaela Ionescu-Bujor and Dan G. Cacuci. A comparative review of sensitivity and uncertainty analysis of large-scale systems - I: Deterministic methods. Nuclear Science and Engineering, 147(3):189-203, 2004.

[14] P.-Y. Lagrée. An inverse technique to deduce the elasticity of a large artery. Eur. Phys. J./AP, 9:153-163, 2000.

[15] Emmanuel Laporte and Patrick Le Tallec. Numerical methods in sensitivity analysis and shape optimization. Modeling and Simulation in Science, Engineering, \& Technology. Birkhäuser Boston Inc., Boston, MA, 2003. With 1 CD-ROM (Unix, Macintosh).

[16] Francesco Migliavacca, Gabriele Dubini, Giancarlo Pennati, Riccardo Pietrabissa, Roberto Fumero, Tain-Yen Hsia, and Marc R. de Leval. Computational model of the fluid dynamics in systemic-to-pulmonary shunts. J. Biomech., 33(5):549-557, 2000.

[17] Francesco Migliavacca, Giancarlo Pennati, Gabriele Dubini, Roberto Fumero, Riccardo Pietrabissa, Gonzalo Urcelay, Edward L. Bove, Tain-Yen Hsia, and Marc R. de Leval. Modeling of the norwood circulation: effects of shunt size, vascular resistances, and heart rate. Am. J. Physiol. Heart. Circ. Physiol., 280:H2076-H2086, 2001.

[18] A. Quarteroni and A. Valli. Numerical Approximation of Partial Differential Equations. Springer-Verlag, Berlin, 1994.

[19] Alfio Quarteroni and Luca Formaggia. Mathematical modelling and numerical simulation of the cardiovascular system. In Handbook of numerical analysis. Vol. XII, Handb. Numer. Anal., XII, pages 3-127. North-Holland, Amsterdam, 2004.

[20] S. J. Sherwin, L. Formaggia, J. Peiró, and V. Franke. Computational modelling of 1D blood flow with variable mechanical properties and its application to the simulation of wave propagation in the human arterial system. Internat. J. Numer. Methods Fluids, 43(6-7):673-700, 2003. ECCOMAS Computational Fluid Dynamics Conference, Part II (Swansea, 2001).

[21] N. P. Smith, A. J. Pullan, and P. J. Hunter. An anatomically based model of transient coronary blood flow in the heart. SIAM J. Appl. Math., 62(3):990-1018 (electronic), 2001/02. 